\title{
An Evaluation of Sensor and Data Fusion Technologies for Application within an Integrated Base Defence System
}

\author{
James Spillings ${ }^{1}$ and Peter Burke. ${ }^{2}$ \\ General Dynamics UK Ltd, Bryn Brithdir, Oakdale Business Park, Oakdale, South Wales, NP12 4AA, United \\ Kingdom \\ and \\ Antonios Tsourdos ${ }^{3}$, Peter Silson ${ }^{4}$ and Brian White ${ }^{5}$ \\ Department of Informatics \& Sensors, Cranfield Defence \& Security, Cranfield University, Defence Academy of the \\ United Kingdom, Shrivenham, Swindon SN6 8LA, United Kingdom
}

\begin{abstract}
The purpose of this paper is to communicate the technical issues involved in integrating data fusion technologies in the development of an integrated base defence system. Options are presented, with possible alternatives, when selecting the sensor systems to support specific mission goals. The document then provides a proposed candidate system architecture that integrates the independent systems to support the commander's information needs.
\end{abstract}

\section{Introduction}

The following is an excerpt from a recent report from Afghanistan that clearly highlights the changing face of modern warfare.

"The tempo of Taliban attacks on the compound and the Fire Support Base Hill may have become less than that experienced by the Gurkhas but most nights are disturbed by grenades being thrown from sangars and random incoming 7.62 rounds fired by Taliban snipers. (Anyone firing single shots is a sniper!) There are in excess of 100 identified sniper positions all around the compound and hill top and those on duty in the sangars (4 in each) have to keep watching them, which is especially tiring at night with CWS."

The Main Operating Base forms a permanent presence within an area of interest and serves as the primary staging area for tactical operations conducted by UK forces. The site is traditionally composed of permanent structures i.e. airfield, hospital and vehicle repair facilities and also temporary structures that together house and support thousands of UK personnel and their equipment. Recent experience from operations TELIC and HERRICK have highlighted the increasing number of attacks against UK facilities and personnel at these sites. The representative threats to UK personnel are attributed to local militia employing sniper fire; indirect fires composed of mortar and rocket attack and improvised explosive devices (IEDs). In the presence of these threats personnel must continue to secure the base perimeter and the surrounding area from continued intrusion and attack by local militia.

The traditional means of maintaining a secure base perimeter primarily relied upon mounted and dismounted teams of personnel assigned to patrol and watch in, and around the base perimeter for any potential threats or signs of disturbance. The primary disadvantage of these patrols is the potentially large numbers of personnel required to cover a typically large area, reducing the resources available to the commander. It is also easy for people to switch off and the use of human patrols can become both static and predictable. Larger sites may also employ CCTV

\footnotetext{
${ }^{1}$ Engineer, Department Name, General Dynamics UK Ltd.

${ }^{2}$ Engineer, Department Name, General Dynamics UK Ltd.

${ }^{3}$ Head of Autonomous Systems, Department of Informatics \& Sensors, AIAA Member.

${ }^{4}$ Assistant Professor, Department of Informatics \& Sensors.

${ }^{5}$ Professor Emeritus, Department of Informatics \& Sensors, AIAA Member..
}

1

American Institute of Aeronautics and Astronautics 
systems in an attempt to monitor on-site activity; however such systems offer limited coverage and require human operators to continuously monitor the current situation and raise the alarm in response to any identified threat.

\section{A. Problem Definition}

There is a requirement for an integrated suite of sensors and countermeasures to provide a complete system capable of enhancing the defence of an operational base supporting tactical operations. The integrated system must be capable of acquiring sufficiently accurate data concerning the following threats to support the commander in selecting the appropriate response to a confirmed threat. The following diagram, Figure I-1, depicts the operational scenario.

The base location is deemed to be the worst case scenario in future Middle East operations. To the west of the camp are some hills that could potentially provide cover for snipers and mortar attacks. To the south of the camp is a city, one that has recently been secured as a safe zone; however there are still potential threats from insurgents. The likelihood of a threat from the various regions is indicated by the colour that shrouds the area. The mountains and the front of the town are deemed to be the areas where an attack is most likely to be formed. The yellow zone, although containing the secured town, can also pose a threat and must be monitored at all times. It is likely

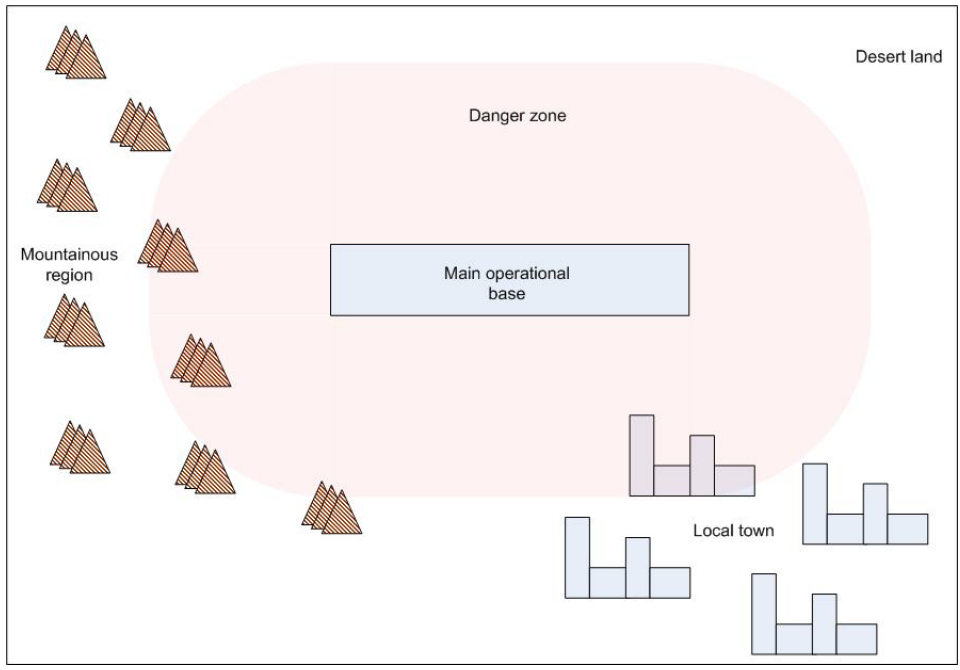

Figure I-1 High-level Operational Scenario that an entrance will be sited along the wall facing the town. A more detailed representation of the base will be provided in a later section.

The specific location of the base has been chosen using a set of high-level requirements; the foremost being room for a landing strip. In this scenario the landing strip is actually the local town's airfield. By securing this UK forces can control the local airspace and regulate the flow of airborne traffic into and out of the town.

\section{B. Issues in Applying Data Fusion Technologies}

Multi-sensor data fusion, or distributed sensing, has been developed primarily in response to a need to solve a diverse set of problems in the military domain; the common characteristic amongst all applications being the requirement to improve the estimation of location and identity of entities, which may not be feasible from an individual sensor alone. The benefits of data fusion include increased spatial coverage, extended temporal coverage, improved detection, reduced uncertainty in inferences, improved system reliability, improved Operational performance.

Implementing systems employing data fusion techniques is not a simple process and the quantitative improvements in system performance that result from employing multiple sensors must consider a number of issues ${ }^{6}$;

- What are the outputs of the data fusion system? Are the system outputs directly related to input data (e.g. the target state vector) or are more complex patterns or inferences sought?

- What data is potentially observable? What are the physical phenomena available for observation e.g. electromagnetic, chemical, acoustic, seismic?

- What is the sensing environment? Does interference, noise or ECM degrade sensor observations?

\footnotetext{
${ }^{6}$ Mathematical Techniques in Multisensor Data Fusion
} 
- What sensors are available? What are the types of sensors? Are they active, passive or guided by the fusion system?

- What are the observed data types and their associated rates?

- What is the timeline for the decision process? That is, does the time an inference is required span milliseconds, seconds, minutes, hours or days?

A key component of the system analysis process is to consider how these factors impact sensors, both individually and in concert, in the pursuit of inferences sought by the data fusion system. Strictly speaking there is no such thing as a data fusion system; rather data fusion is a derived required in the development of a complex system. Our approach to evaluating the application of data fusion techniques in this investigation follows a general systems engineering process ${ }^{7}$ that incorporates common guidelines [Hall, course books] with respect to data fusion such as sensor selection, architecture selection and algorithm selection.

\section{Initial Analysis}

Prior to embarking on the specific design aspects of the architecture and solution some initial analysis needs to be completed. This section introduces the base in more detail and subsequently identifies the threats that the base is exposed to. Once the threats have been characterised, the counter-threats to be employed can be explored. The highlevel functional requirements for the base defence system are developed and the associated constraints imposed upon them are highlighted.

\section{Concept Development}

The following diagram, Figure II-1, provides a more detailed illustration of the generic operational base and its surrounding environment. The base covers an area of $5 \mathrm{~km}^{2}$ and includes a landing strip and a chain of related buildings, a number of which have been commandeered by UK forces to serve as their command HQ. The site is surrounded by a high-level

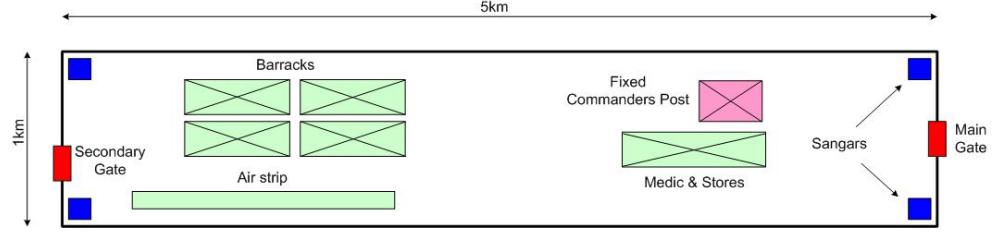

Figure II-1 Detailed layout of the operational base. fence, with additional high walls built at selected points around the site in order to reduce visibility into the compound. Entry into and out of base is controlled via two main checkpoints; primary and secondary exits fortified with gate barriers with a number of sangars overlooking the check points and the approaching roads. The commander has available to him both mounted and dismounted teams of personnel that may be assigned to patrol on-site and the surrounding area. The command HQ established at the base serves as the main $\mathrm{C} 2$ centre for a Brigade-level force deployed to that area. The command HQ can be considered to be the location from which the commander will coordinate a response to events in and around the base.

\section{Threat Characterisation}

Before considering any counter threat system the main enemy threats need to be identified. As the location of the base is within close attack range of the town and also the mountainous region the initial threats will undoubtedly come from these regions. As such at this time there will be no consideration paid to the long range threats such as air assaults or long range tank attacks; these will be discussed, from a high-level perspective later in the document. The following subsections describe the identified threats and provide information on the areas where the threats are vulnerable; the areas where the counter threat systems can exploit them.

\section{Sniper $\backslash$ Gun Fire}

Snipers, predominantly working alone or in pairs (one is the shooter and one is the spotter) are masters at hiding. They have the ability to blend into the scenery making them appear almost invisible and as such very hard to find. The sniper will usually be equipped with a powerful and highly accurate rifle mainly used for targets up to approximately 2000 meters. Snipers, although very well camouflaged have one main attribute that they cannot hide; the sniper rifle's sight. In order for the sniper to shoot a target they must be able to see that target requiring both the

\footnotetext{
${ }^{7}$ International Council on System Engineering - $\frac{\text { http://www.incose.org/ }}{3}$
} 
rifle muzzle and sight to be visible. The muzzle can be hidden however the reflectiveness of the sight is the main weakness. The other attribute of snipers is the acoustics from the weapon being discharged. This, although muffled by snipers cannot be completely muted. Once a round has been discharged the sniper can leave the scene.

\section{Mortars \& Rockets}

Mortars, simple by design and operation, have been adapted and embraced by insurgent groups. The mortar is a common infantry support weapon, man portable with an effective range up to 2500 meters; this is dependant on the angle of launch and environmental conditions. The lob style firing means that it can be hidden in pre-made trenches or from behind other obstacles. Rockets, holding much of the attractiveness of mortars such as the mobility and destructiveness, are fired from the shoulder. The range of the rocket is generally in the region of $400-500$ meters though home made ones may not travel so far. The Rocket Propelled Grenade (RPG-7) is a direct-fire weapon used by insurgents although the range, with moderate accuracy, is limited to about 90 meters. The RPG may be used to provide a limited bombardment capability from a range of some 900 metres. Although all of the above are effective methods for attack the mortar causes the most concern. This is mainly due to the restricted range of rockets and exposure of the operator when firing. Although the mortar does have many beneficial attributes it is also easy to detect and eliminate. This is mainly due to the lob action of the munitions combined with the acoustic disturbance caused by the round being discharged.

\section{Vehicles}

An enemy vehicle itself poses little threat to the base; it is what is being carried that will cause the damage. All of the above mentioned threats can be easily transported in a vehicle no larger than a standard military land rover. All vehicles that are likely to be employed by insurgents will be generally of civilian ilk.

\section{Personnel}

Military personnel, which we use in this context to refer to enemy infantry and militia forces, represent the largest threat to the security of base. They can be indistinguishable from one another meaning militants can integrate with civilians and become invisible. The main focus of people in this paper is those that have intent and approach the base in such a manner that they can be identified as being an aggressor.

\section{Threat Summary}

The following table provides a matrix summary of the associated sensors that could be used for each threat.

\begin{tabular}{|l|l|l|l|l|l|}
\hline & Acoustic & Infra red & Electro optic & Radar & Laser \\
\hline Sniper & $\checkmark$ & $\checkmark$ & & & $\checkmark$ \\
\hline Mortar $\backslash$ rockets & $\checkmark$ & $\checkmark$ & & $\checkmark$ & \\
\hline Vehicles & & $\checkmark$ & $\checkmark$ & $\checkmark$ & \\
\hline Personnel & & $\checkmark$ & $\checkmark$ & $\checkmark$ & \\
\hline
\end{tabular}

Table II-1 Summary of threats

\section{Requirements Analysis}

This section focuses on the high-level requirements of the overall system. These requirements are the basis for the design of the system architecture and eventual system solution. Identified are the main counter threat areas based on the threats that were identified in previous sections accompanied by a description of their high-level requirements. The table below, Table III-1, identifies the high level system requirements.

\begin{tabular}{|l|l|}
\hline 1 & $\begin{array}{l}\text { Sensor systems that are incorporated into the base defence system and interface with the Bowman } \\
\text { C4I system to utilise its capabilities must comply with the specific requirements outlined in the } \\
\text { Supplementary Requirements E section. }\end{array}$ \\
\hline 2 & The system must detect hostile sniper fire rapidly. \\
\hline 3 & $\begin{array}{l}\text { The system must be capable of locating sniper fire in azimuth, elevation and range out to a distance } \\
\text { of } 1.6 \mathrm{~km} .\end{array}$ \\
\hline 4 & $\begin{array}{l}\text { The system must display the estimated range and bearing to the sniper from the operator's current } \\
\text { location. }\end{array}$ \\
\hline 5 & The system must be capable of being calibrated to enhance the detection of specific calibre of bullet \\
\hline
\end{tabular}




\begin{tabular}{|l|l|}
\hline & as mission objectives require. \\
\hline 6 & $\begin{array}{l}\text { The system must be capable of detecting and tracking indirect-fire in azimuth, elevation and range } \\
\text { out to a distance of } 6 \mathrm{~km} .\end{array}$ \\
\hline 7 & The system must determine the point of origin for mortar and rocket fire. \\
\hline 8 & $\begin{array}{l}\text { The system must display the estimated location and range to the point of origin of the indirect fire } \\
\text { to the human user. }\end{array}$ \\
\hline 10 & $\begin{array}{l}\text { The system must be capable of intercepting confirmed mortar and rocket fire in adverse weather } \\
\text { conditions including rain, fog and hail. }\end{array}$ \\
\hline 11 & $\begin{array}{l}\text { The system must be capable of detecting and tracking indirect-fire in azimuth, elevation and range } \\
\text { out to a distance of } 6 \mathrm{~km} \text {. }\end{array}$ \\
\hline 12 & The system must provide effective $360^{\circ}$ surveillance beyond the surrounding perimeter. \\
\hline 13 & The system must detect men and vehicles out an effective range of 5km beyond the base perimeter. \\
\hline 14 & The system must display the estimated location and range to a potential target to a human operator. \\
\hline 15 & $\begin{array}{l}\text { The system must identify vehicle and personnel surrounding the perimeter from an effective range } \\
\text { of } 4 \text { km. }\end{array}$ \\
\hline 16 & The system must provide an effective day and night surveillance capability. \\
\hline 17 & $\begin{array}{l}\text { The system must provide an effective surveillance capability in varying environmental conditions } \\
\text { that include rain, fog and the contrasting climates of Europe and central Asia. }\end{array}$ \\
\hline 18 & $\begin{array}{l}\text { The integrated system must reduce the number of personnel required to continuously maintain a } \\
\text { secure perimeter in and around the base. }\end{array}$ \\
\hline
\end{tabular}

Table III-1 functional requirements for the integrated base defence system.

Our approach to the development of a preliminary design of the integrated defence system is to subdivide the system into a number of independent components or subsystems. The complete system is decomposed by function, where each subsystem is designed to address specific mission goals and engage specific threats and is therefore capable of being employed independently. The key roles and responsibilities of each subsystem are described in the following subsections.

\section{Sniper Detection System}

The sniper detection system is responsible for estimating the location of sniper and small arms fire surrounding the perimeter of the base. The system must potentially deal with multiple targets at varying rates of fire and must inform a human operator of the firing location of the sniper. The system should be capable of being deployed at both fixed sites and on mobile units assigned to patrol the area surrounding the base.

\section{Indirect Fire Countermeasure System}

The indirect-fire countermeasure system is responsible for detecting and tracking incoming mortar and RPG fire and must provide an estimate of the firing location of the munitions in the area surrounding the base. The subsystem must intercept confirmed mortar and RPG fire directed at the site under varying weather conditions and must inform the human operator of the firing location and estimated impact point of the munitions.

\section{Perimeter Surveillance System}

The perimeter surveillance system is envisaged to provide a wide-area, all-weather surveillance capability that may be leveraged to detect and identify men \& vehicles that are within a confirmed distance from the base perimeter. This subsystem provides enhanced awareness of general activity at short to medium ranges around the base perimeter and enables the commander to deter or intercept confirmed threats using available resources.

\section{Tactical C4I System}

A command and control (C2) system is a complex integration of communications and computing infrastructure and software services. A C2 system is a typical real-time system. The C2 system is core to the execution of tactical operations and provides timely information to the commander to allow the right decision to be made at the right time.

\section{E. Supplementary Requirements}

The British Army employs a tactical communications system that provides secure voice \& data services to dismounted soldiers, vehicles and command HQs up to Division-level. The system is comprised of HF, VHF and 
UHF radio sets, supporting integrated GPS receivers and frequency-hoping spread spectrum techniques to reduce the effects of Electronic Counter Measures (ECM). The underlying network architecture encompasses open, standardsbased communications protocols that include TCP/IP for relaying data between network nodes. These technologies work in concert to form a Mobile Ad-hoc Network (MANET) that supports a number of software services. Vehicular and static users i.e. those at a command HQ level are provided with computer terminals to provide tactical situational awareness. Each terminal employs a Geographical Information System (GIS) that supports multiple mapping formats and allows the visualisation of geo-referenced data, such as a unit's current location and those of surrounding blue-forces. To promote a coherent view of the evolving dynamic battlefield, the system supports the NATO JC3IEDM specification, a data and information exchange standard that specifies the minimum amount of data to be exchanged between NATO allies in the $\mathrm{C} 2$ environment. The data model describes all objects of interest in the sphere of operations e.g. organisations, persons, equipment, geographic features and their attributes. The commander will utilise the situational awareness services and communications capabilities provided to him via this tactical communications network to execute command and control. Subordinate units assigned to base protection, including mounted and dismounted units, will have access to this system. The tactical C4I system specifies a number of system-level interfaces that external systems must comply with in order utilise the services it provides. These system-level interfaces define electrical, mechanical and functional behaviours. Where external sensor systems are to be integrated with the tactical C4I system consideration must be given to the interface and physical constrains.

Interface Constraints:

- The HF and VHF radios employed by the tactical C4I system provide a narrowband, high latency data channel with suitable communication ranges for this project.

- The UHF radio subsystem employed by the tactical C4I system is a line-of-sight, wide-area communications network providing a medium capacity data channel.

- The tactical C4I system supports IP-based communications for data transfer.

- The GIS system employs a set datum, map format and a limited number of projections for visualising geospatial data.

- Situational Awareness data will be distributed between the UK and its allies in accordance to the JC3IEDM specification.

Physical Constraints: Where a mounted unit is to be integrated into the system, the free-space available within the unit is extremely constrained. Consideration must therefore be given as to the dimensions, weight and cooling requirements of any hardware that will be installed onto the unit as part of the integrated base defence system.

\section{Counter system analysis \& design}

The following subsections detail the counter system designs. It is beyond the scope of this document to fully design the sensors themselves and only attributes of the sensors will be mentioned, where relevant.

\section{F. Sniper Detection System Analysis}

The operating base is likely to be exposed to sniper attacks both for personnel on the base as well as for any mobile crews that are to perform a recce task. Although the availability of high powered sniper rifles is low for the expected aggressor the weapons they are using are still capable of causing harm from range. In Iraq, the infamous Juba ${ }^{8}$ uses the Tabuk sniper rifle with standard Kalashnikov rounds and probably has a range of 500-600 metres. It has been described as more of a marksman's rifle than a sniper rifle, which is designed to be accurate beyond 800 meters. The snipers are able to hide in local buildings and integrate into the local populous seamlessly and as such can be difficult to detect. The rounds are fired and the assailant flees almost immediately. It is understood from various news reports and videos posted on the internet that the snipers are targeting personnel in both stationary and mobile positions showing that nobody is impervious to the attacks. The requirements identified in Table III-1 provide an initial template for the design of the sniper detection system. As mentioned previously this system will be required to estimate the location of the sniper autonomously while providing as much information to the human operator. The final general requirement is that the system must be able to be deployed in a static and mobile configuration.

\section{Detailed Requirements}

The table below, Table IV-1, contains the detailed requirements for the sniper detection system. When deciding the requirements that have been laid down below the following aspects have to be considered:

\footnotetext{
${ }^{8}$ Routers report on the $29^{\text {th }}$ October 2006
} 
- Where is the threat? This is solved with the use of a relevant sensor. In the case of sniper detection there are multiple methods that can be used; these will be discussed in a later sub section.

- Where is it going to? This is not direct issue when considering snipers as they tend to be stationary when firing. However obtaining the information of the snipers location is vital and must be done very quickly.

- What is it? This is mainly concerning the identification of the object being sensed however it is already known that a sniper is the target and its characteristics understood.

- Is it a threat? This information is obtained through the analysis of the data provided by the sensor. It can be assumed that all snipers are threats; however the last aspect is vital.

- What action is required? Is it suitable to counter the sniper immediately with gun fire or can the threat be neutralised in another way; perhaps by guiding another sensor on to it to in order to obtain more information.

\begin{tabular}{|l|l|}
\hline Requirement Title & Requirement Description \\
\hline Time & Provide all required information within one second of the shot \\
\hline Shot miss & Must be able to reliably detect a missed shot up to 20 meters away \\
\hline Range & Must be able to reliably localise a shooter up to 500 meter away \\
\hline Accuracy & $\begin{array}{r}\text { Must be able to localise the target to within: } \\
\pm 15 \text { degrees accuracy in azimuth } \\
\pm 10 \text { meters positional localisation } \\
\pm 10 \text { degrees accuracy in elevation }\end{array}$ \\
\hline Environmental & $\begin{array}{l}\text { Must be able to withstand both humid and arid conditions, with temperatures up } \\
\text { to 50 degrees Celsius. }\end{array}$ \\
\hline Mobility & $\begin{array}{l}\text { Must be able to be used in both a static and mobile configuration on a vehicle } \\
\text { travelling up to 50 miles per hour. }\end{array}$ \\
\hline Alert & Must provide both visual and audible alerts to the human user. \\
\hline Data & $\begin{array}{l}\text { Must be able to provide accurate azimuth and elevation data to enable another } \\
\text { sensor to be guided onto the target. }\end{array}$ \\
\hline Replacements & The unit must be field replaceable. \\
\hline
\end{tabular}

Table IV-1 Detailed sniper requirements

\section{Designing the System}

There are various methods of detecting a sniper, each with there associated benefits and issues. The following subsections identify the various techniques that can be employed (at the sensor level) and highlight the final technique that will be used in this system.

\section{Temporal Pattern Recognition}

This technique ${ }^{9}$ effectively employs neural networks that have been trained to identify the specific sound created when a round is shot from a sniper rifle. Once the sound has been recognised as a gunshot, as opposed to other similar noises that are created in the immediate battlespace, triangulation of that noise can be performed and the target subsequently located. Training neural networks can be a cumbersome and often error prone process and as such would not be deemed to be ideal for this system.

\section{Optical Localisation}

Optical Localisation ${ }^{10}$ uses electro-optical (EO) sensors to detect muzzle flash. It can also detect the heat the bullet dissipates as it travels through the air. This system relies on the being a good muzzle flash and the camera being focused on the sniper at the time of fire; it is likely that this would happen. As well as this muzzle suppression equipment can also be used to hide the muzzle flash. The use of an EO sensor would be valuable for gathering more information of the target once it has been localised.

\footnotetext{
${ }^{9}$ http://www.safetydynamics.net/

${ }^{10}$ http://www.radiancetech.com/products/weaponwatch.htm
} 


\section{Seismological Techniques}

This technique ${ }^{11}$ employs similar methods used when detecting earthquakes and tremors. It relies on the gun shot occurring within an array of microphones and the tremors being sampled at a high rate. This is fairly accurate though would require a substantial number of arrays to cover the size of area being considered here.

\section{Acoustic Localisation}

Acoustic localisation ${ }^{12}$ involves the analysis of the shock wave created by the round as it flies through the air and the noise emitted by the weapon as the round is fired. By looking at these two attributes it is possible to accurately locate the sniper providing the target bearing, elevation and distance to the target. Another encouraging factor is that the array of microphones employed can be fairly small and the round need not fly through the array in order for a detection to occur. This is the technique that will be employed in the design of the sniper detection system.

\section{Secondary Assets \& Data Requirements}

Once the system detects a potential target the data needs to be used in an effective manner, however what will the data be and how will it impact on the communications systems being employed to disseminate the data. The data that will be provided by the sniper detection system will include the targets azimuth, range and elevation. There may be some potential for an extension to the system to enable it to ascertain the make of weapon being fired however at this time this functionality is not under consideration. The data produced is relatively small in size and should not require a high bandwidth link. The system modules will be connected via hard wire connections as this is logically the most sensible method for connecting the system together; all components of the system are to be co-located. This data may be required to be passed to a co-located secondary sensor, such as an EO sensor. This data could cue this sensor to enable more data gathering to occur. The data may also be passed to other personnel; the G2 cell (Intelligence and Security) to enable a picture to be constructed of the assault or the Royal Artillery to enable munitions to be deployed on the target. As the data to be transmitted throughout the base is nominal it is deemed that there will be little impact on the communications network; as such the location of the sensors is not an issue as a low bandwidth wireless link would suffice. If a complimentary sensor is employed and data from a live video stream is required then there will be an impact on the network. For the most there will be little requirement for live streaming of data to be passed to the $\mathrm{C} 2$ tent however should the commander request a live feed then it can be expected that a $256 \times 256$ image at 2 frames per second would require approximately $15 \mathrm{kbps}$ of bandwidth. If a collocated sensor is to be used then it will need to be fit for purpose and cannot simply be a generic sensor. There are many standard visual sensors that can be employed however, should the use of an IR sensor be required then it will need to be specified correctly. The sensor will be using looking at the $10 \mu$ waveband and as such will have a pixel size of approximately $25 \mu$. Considering a 128x128 2D array sensor and an expected target size of about 1 meter the focal length is approximately $10 \mathrm{~cm}$. With this in mind the overall field of view of (FOV) the camera will be bout $25^{\circ}$. In order to detect the target and in fact to recognise the target the number of pixels needs to be greater than 100; the more the better. As such the number of pixels could be increased to 256 . This would effectively increase the FOV to about $100^{\circ}$. The ultimate trade off is cost in this situation. The size of the camera can, within reason, be quite big to accommodate the other, more important requirements. It can also be a liquid cooled camera as it will be collocated with the acoustic sensor; likely positioned at an outlook post.

\section{Sensor System Architecture}

The final solution will employ acoustic localisation. There is subsequently a requirement for an array of microphones able to detect frequencies in the range $1 \mathrm{KHz}-10 \mathrm{KHz}$ (standard frequency range of a gun shot). The signals must then be passed to a processing unit where, through the use of suitable algorithms, the location, azimuth and bearing of the sniper must be presented to the user through the use of visual and audible alerts. This data must also be presented to a secondary sensor, where available, to enable that sensor to analyse the target further, if required. The following diagram, Figure IV-1, is the basic architecture of the system. As can be seen the acoustic aspect of the system is relatively simple in design and has a small form factor allowing it to be located on the roof of a watch tower or even off the back of a HWMMV, or equivalent vehicle. The complete architecture shown in Figure IV-1 contains the links from the acoustic sensor through to the secondary sensor and the rapid reaction weapon. All of these logically link to the bases' communications system and as such will be able to pass data to the $\mathrm{C} 2$ terminals as required. The specified system need not be designed if there is an equivalent system Commercial Off-The-Shelf

\footnotetext{
${ }^{11} \mathrm{http} / / / \mathrm{jclahr} . c 0 m / \mathrm{science} / \mathrm{psn} / \mathrm{gunshots} / \mathrm{of} 93221 / \mathrm{index} \cdot \mathrm{html}$

12 http://www.bbn.com/products_and_services/boomerang/
} 
system available. Research has identified the Boomerang ${ }^{13}$ system, designed by BBN Technologies ${ }^{14}$. Although the

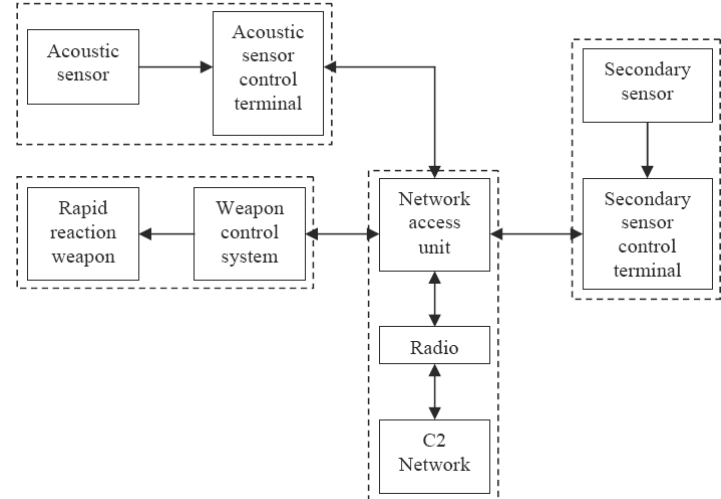

Figure IV-1 Sniper detection system architecture

above identified system ha been designed for use on a moving vehicle there is no perceivable reason to suggest it cannot be employed in a static configuration. There would however need to be a small amount of work required in order to extract the information requiredto guide a secondary senor to the target location.

\section{G. Indirect Fires Countermeasure Subsystem Analysis}

\section{Defence against Mortar and Rocket Attack}

The operating base is subject to mortar and rocket attack from local militia forces, where the environment favours attack from the nearby town and surrounding hillside. The militia prefer to avoid direct confrontation with security patrols and, due to their lack of conventional support in the form of mechanised platforms and artillery, rely upon the man-portable $60 \mathrm{~mm}$ and $81 \mathrm{~mm}$ mortar as their primary infantry support weapon. In addition to the mortar, $107 \mathrm{~mm}$ rockets have been known to be used against UK facilities and provide the enemy with a medium range, indirect fire capability.

\begin{tabular}{|l|l|l|l|}
\hline & Effective Range & Mean Velocity & Estimated Rate of Fire (rounds per minute) \\
\hline $60 \mathrm{~mm}$ Mortar & $3 \mathrm{~km}$ & $200 \mathrm{~ms}^{-1}$ & 20 \\
\hline $81 \mathrm{~mm}$ Mortar & $6 \mathrm{~km}$ & $300 \mathrm{~ms}^{-1}$ & 15 \\
\hline $107 \mathrm{~mm}$ Rocket & $5 \mathrm{~km}$ & $330 \mathrm{~ms}^{-1}$ & unknown \\
\hline
\end{tabular}

Table IV-2 threat profile for mortar and rocket fire

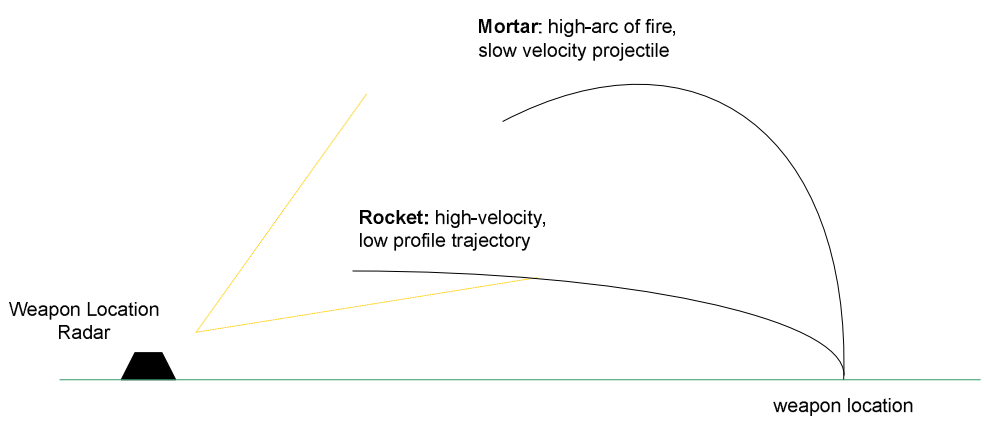

Figure IV-2 flight characteristics of mortar and rocket fire
Mortar and rocket fire are different in nature and require separate consideration in the development of a defence support system. The mortar is distinguished by its relatively low velocity and parabolic arc of fire, with rocket fire considered to follow a high-velocity, low elevation path to the target (Figure 1). The use of either weapon can render the operator highly vulnerable due to the potential to locate the firing point from the initial muzzle flash and acoustic signature of the weapon launch. Note that the

mortar's low thermal signature, in comparison to the rocket, is a further distinguishing factor between the weapons systems. Given knowledge of the target weapon systems and their defining characteristics, we expand the functional requirements of the Indirect Fire Countermeasure system from the original high-level requirements presented in 2.3 as follows:

\begin{tabular}{|l|l|}
\hline 1 & $\begin{array}{l}\text { The system must be capable of detecting and tracking mortar fire in azimuth, elevation and range out to } \\
\text { a distance of } 6 \mathrm{~km} .\end{array}$ \\
\hline 2 & $\begin{array}{l}\text { The system must be capable of detecting and tracking rocket fire in azimuth, elevation and range out to a } \\
\text { distance of } 5 \mathrm{~km} .\end{array}$ \\
\hline 3 & The system must determine the point of origin for mortar and rocket fire to an accuracy of $100 \mathrm{~m}$ at $5 \mathrm{~km}$. \\
\hline 4 & The system must display the estimated point of origin of the indirect fire to the human user. \\
\hline 5 & The system must display the range and bearing to the point of origin of the indirect fire from the human \\
\hline
\end{tabular}

${ }^{13} \mathrm{http}: / /$ www.bbn.com/products_and_services/boomerang/

${ }^{14}$ http://www.bbn.com/ 


\begin{tabular}{|l|l|}
\hline & user's current location. \\
\hline 6 & $\begin{array}{l}\text { The system must be capable of intercepting multiple confirmed mortar and rocket munitions in adverse } \\
\text { weather conditions including rain, fog and hail, down to a minimum distance of } 200 \mathrm{~m} .\end{array}$ \\
\hline 7 & $\begin{array}{l}\text { The system must determine the point of impact of incoming mortar and rocket fire and display the } \\
\text { estimated point of impact to the human user. }\end{array}$ \\
\hline 8 & The system must store the estimated point of origin and point of impact for all confirmed attacks. \\
\hline 9 & $\begin{array}{l}\text { The system must issue an audible alarm at areas around the base that are determined to contain the point } \\
\text { of impact of incoming munitions so as allow base personnel to time to disperse. }\end{array}$ \\
\hline
\end{tabular}

Table IV-3 expanded requirements for Indirect Fires Countermeasure system

The system requirements in Table 2 will be aligned against the NATO 'Defence Against Mortar Attack' (DAMA ${ }^{15}$ ) initiative:

- Detect: detect when and where a mortar or rocket will be fired

- Warn: estimate the likely point of weapon impact and provide warning to personnel

- Intercept: engage confirmed mortar and rocket fire and neutralise the weapon in-flight.

- Command and Control: provide information to the commander to enable him to deter further threat from mortar and rocket fire and optimise the use of the Indirect Fire Countermeasure system.

\section{H. Detect}

\section{Candidate Sensor Technologies}

A review of existing or in-service counter-artillery systems reveals the use of acoustics ${ }^{16}$ and $\operatorname{radar}^{17}$ as the primary sensor technologies for weapon location. Acoustic weapon location offers a NLOS, passive detection system that has proven to be capable of locating artillery fire beyond $20 \mathrm{~km}$ in range and is capable of observing the large areas needed for traditional counter-artillery operations. Acoustic sensors may also operate over a relatively wide bandwidth, capable of searching across a wide range of frequencies to classify various weapon systems. However, the maximum range and accuracy of acoustic systems is weather dependant due to the effect of winds and temperature variation. Current acoustic weapon location systems, such as the HALO system from Selex, rely upon deploying a series of networked sensor nodes over a wide area, making the system time-consuming to effectively deploy. In contrast, radar provides an all-weather, LOS capability that can be made mobile and is thus quick to deploy. Radar is an active system and provides high-data and target acquisition rates relative to acoustic systems, where the high-data rate can be leveraged to provide accurate weapon identification and tracking. The active nature of radar can render the system vulnerable to ECM and direction-finding, however these disadvantages do not outweigh the potential benefits of using radar for weapon location. In considering the applicable technologies for detecting \& tracking incoming mortar and rocket munitions, radar is considered to be the most suitable sensor technology for the following reasons:

- The threat from mortar and rocket attack is from relatively close range, requiring a highly responsive system capable of detecting and tracking incoming fire, with minimal delay, in all weathers.

- Acoustic weapon location, although effective, is better suited to missions requiring wide area coverage where enemy weapon systems, such as conventional artillery, are located further to the rear.

- The use of acoustic sensors prevents tracking of munitions in-flight and thus cannot support the requirement to intercept indirect fire targeted at the base.

20. Rocket and Mortar Weapon Location Radar

A review of current weapon location radar systems such as the American AN/TPQ-36, 37 (Firefinder family) and the European COBRA and ARTHUR illustrate the change in the nature of the envisaged threat today compared to the threat such systems were designed to counter. Traditional weapon location radars provide a medium to long range $(10 \mathrm{~km}-25 \mathrm{~km})$ artillery and rocket fire location capability. Such systems typically scan a sector $90^{\circ}$ in azimuth for incoming fire, requiring the radar be mechanically steered to observe the required area to provide full $360^{\circ}$ coverage. These systems are also large and demand significant power to detect artillery and rocket fire at long range, requiring the use of a vehicle to support and transport the radar across the battlefield. In contrast, the current threat is considered to originate from infantry support mortars and rocket fire that are likely to originate from within built-up areas or terrain concealing the operator, such as the valleys, hills and forest close to the operating base. The

\footnotetext{
${ }^{15}$ http://www.nato.int/docu/update/2007/03-march/e0327a.html

16 SELEX S\&AS HALO

${ }^{17}$ Raytheon-THALES AN/TPQ 36/37 FireFinder RADAR, BAE Systems MAMBA System
} 
ability to fire from short range and the ease of use of such weapons imply the operating base may be subjected to a

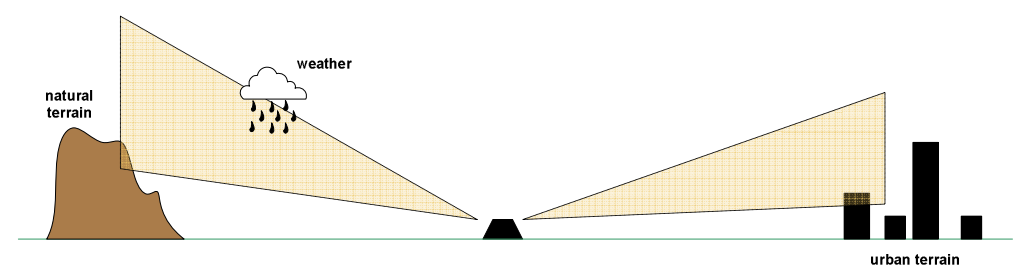

Figure IV-3 Illustration of sources of clutter experienced by radar

high rate of fire, from any angle, with relatively no warning. It is recommended the traditional weapon location radar be replaced by a dedicated short range radar employing an electronically-steered, phased array antenna to provide instantaneous $360^{\circ}$ coverage to detect mortar and rocket fire, similar to the Lightweight Counter Mortar Radar (LCMR) employed by US ground forces.

The use of a phased-array antenna enables an area to be scanned at a high rate and can easily be developed to provide continuous $360^{\circ}$ coverage of the surrounding area, removing the need to mechanically steer the antenna array as in current weapon location radars. The proximity of the nearby town and hillsides will prove to be particularly severe sources of clutter, Figure IV-3, in which the radar must detect and track targets with extremely low radar cross-sectional area. The degree of clutter can be reduced by increasing the antenna's angle of elevation; the accuracy of weapon location however is greatly improved by detecting the incoming projectile as low as possible in its trajectory. There exists therefore a trade-off between clutter reduction and detection performance. The low RCS of mortar and rocket munitions is comparable to that of small birds, thus the radar must be capable of processing and discriminating against a large number of entities in real-time to extract true weapon fire, requiring a large computational capacity. Given the advancements in miniaturisation it is reasonable to assume that sufficient computing resources are available to process the large number of potential targets, and that the final dimensions of the embedded computer are such that the mass and power requirements of the radar are significantly reduced. The mission computer embedded within the radar is designed to perform the following functions:

- Automatic Height Correction: an essential function that must be performed in order to generate accurate estimates for the point of launch (PoL) for the hostile weapon. The radar is capable of accepting Level-1 DTED data to determine the point at which estimated trajectory of the tracked projectile intersects the terrain, thereby correcting for variation in altitude of the firing weapon.

- Target Classification: the ability to arrange all targets tracked by the system into a series of known types: mortar, rocket, light-aircraft, and helicopter

- Zonal Control: the radar may be configured by an operator to restrict the operation of the radar to a discrete set of geographic areas. This would allow the commander to optimise the use of the radar system, directing its attention to areas from which militia are likely or known to launch attacks, or away from areas that are generally considered to pose no threat e.g. toward the direction of the airstrip located on base.

\section{Recommended Radar Specification}

- Electronically steered, phased array antenna

- High Frequency Waveform - enables high gain antenna and narrow beam width, the latter providing high angular resolution.

- Low sidelobes (reduce ground clutter).

- Medium PRF processing

- MTI processing (high sub-clutter visibility)

- Large computational capacity

- Use of Pulse Compression for good range resolution.

- Frequency Agility (to mitigate ECM).

The tracking system enables the trajectory of the target detected by the weapon location radar to be constructed to allow: 
- target discrimination; targets that do not follow the expected trajectory of mortar or rocket fire, or the expected velocity profile typical of mortars and rockets, are quickly rejected

- estimation of the fires point of launch $(\mathrm{PoL})$ and likely point of impact $(\mathrm{PoI})$.

- cueing of mortar and rocket fire countermeasure system to engage incoming projectiles.

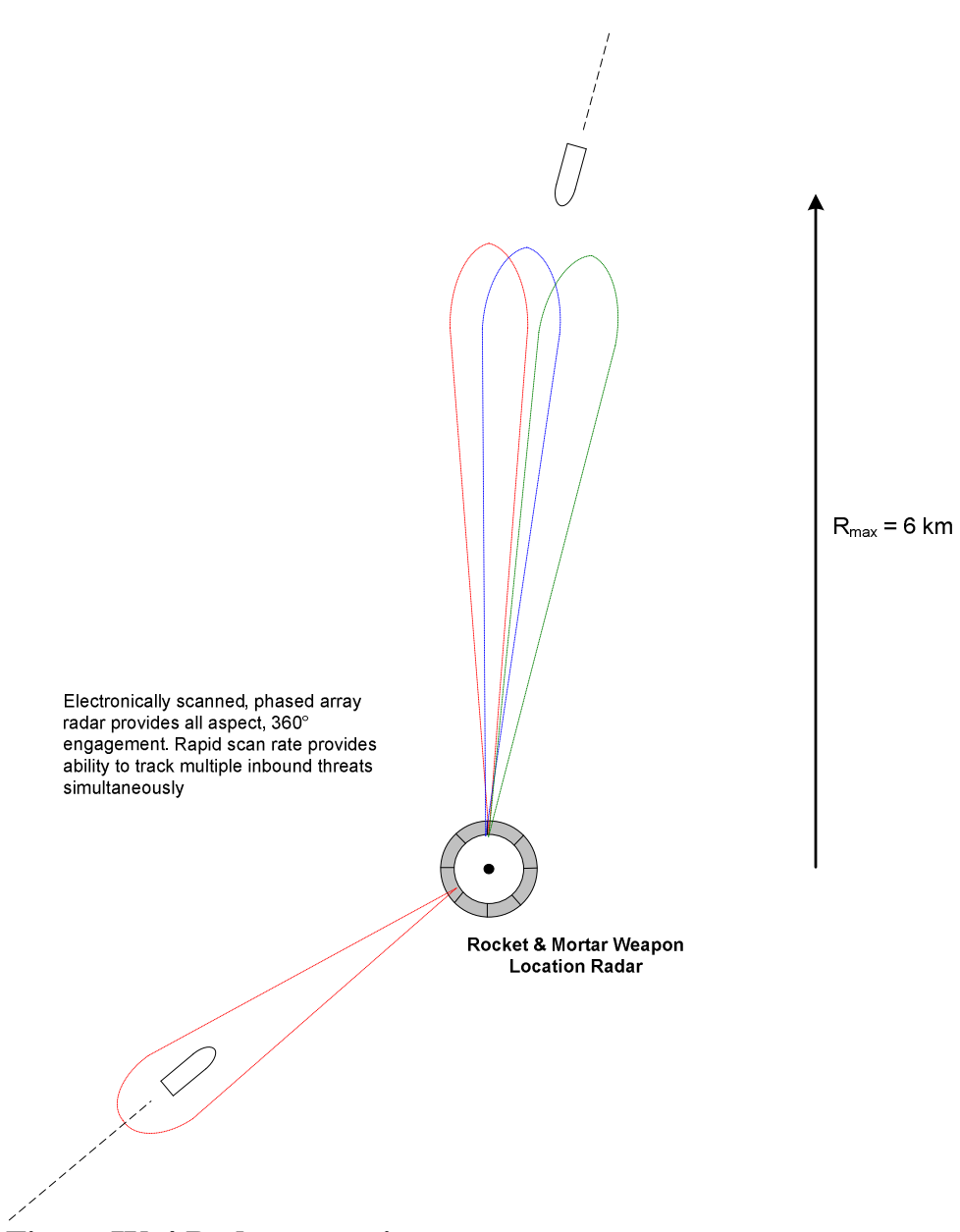

Figure IV-4 Radar operation

Operating Frequency

Minimum Range

Maximum Range

Range Resolution

Angular Resolution

Target Location Error

Antenna Design

Maximum simultaneous target track capacity

Automatic Height Correction

Target Classification

Zonal Control

Integrated Radio Data Link

Capable of being assembled/disassembled by 2 man team in less than 5 minutes.

IEEE L-Band

$700 \mathrm{~m}$

$6.0 \mathrm{~km}$

$15 \mathrm{~m}$

$1.0^{\circ}$

100m@5.0 km

20
Given the high data rate and potential high-false alarm rate of the radar, data association is a primary concern in developing an accurate tracking system. A Probability Data Association (PDA) algorithm has an advantage over other algorithms in that it makes use of all available data in associating a predication with a measurement when constructing a track. Simple gate methods, such as track score and auction algorithms, ignore all measurement data lying outside the gate function, therefore reducing the amount of information available to form a decision. If computational resources allow, the use of PDA algorithm is preferred, or else the use a track score or auction algorithm is an alternative. Following the data-association step in the tracking process, an extended Kalman filter (EKF) is an acceptable algorithm for use in the tracking system due to the non-linear nature of the targets and the Kalman filter's modest computational efficiency, making it suitable for use in an embedded computing environment. The radar initiates a new instance of an EKF for every new track, where the filter employs probabilistic models of real-world disturbances and kinematic models of the mortar \& rocket flight dynamics to estimate the current location of mortar or rocket fire though the air.

electronically scanned phased array

\section{Table IV-4 Final system specification}

American Institute of Aeronautics and Astronautics 


\section{Intercept}

This section addresses the engagement of incoming mortar and artillery fire to prevent their impact within or near the operational base. The interception stage of the indirect fires countermeasure system seeks to maximise both the range at which a confirmed target may be engaged and the probability of neutralising incoming fire via the use of an appropriate weapons system. Candidate weapon systems for engaging incoming mortar and rocket fire include:

- Guided Missiles: their use potentially reduces the required accuracy of the fire-control system since a missile can be configured with a proximity fuse, removing the need to directly hit incoming munitions. A missile-based interception system would however require a suitable amount of preparation before launch and their rate of fire is limited, thus their suitability for engaging numerous, short range munitions is questionable.

- Directed Energy Weapons: refers to the deployment of a laser or focused-microwave beam directed toward incoming munitions, transferring the energy within the beam directly to the round so as to achieve the desired destruction of the mortar or rocket. Such systems would enable a rapidly reduce the time to engage the incoming fire since radiation travels at the speed of light and would also be capable of engaging multiple munitions in quick succession. Research to date has appeared to focus on the development of solid-state lasers for use in close-in weapon defence systems due their safer operation and minimal logistics concerns compared to chemical laser system. The problem however of achieving the required level of power to destroy munitions while minimising thermal blooming, a problem that sees the beam defocus and transfer energy into the surrounding atmosphere, is still under investigation and has prevented the fielding of systems in-theatre.

- Cannon: a simple solution that relies upon firing thousands of rounds of ammunition to achieve the desired probability of success. Cannon-based systems would require careful management of fire in order to ensure they do not expel their entire level of ammunition in a series of short bursts and their effective range is limited to < $2 \mathrm{~km}$. The velocity of a typical $20 \mathrm{~mm}$ round however is close to $1000 \mathrm{~ms}^{-1}$, thus cannon-fire can be expected to intercept incoming munitions with minimal delay.

It is recommended that the indirect fires countermeasure system employ a cannon-based weapon system to intercept confirmed fire in mid-flight. Missile-based systems are considered unsuitable for operation given the envisaged short range from which weapons are launched, and their ability to intercept fire in time is subject to doubt. Directed energy weapons, despite their promise, remain immature at present leaving a cannon based solution as the only remaining option. The following analysis of a cannon-based, close-in weapon system (CIWS) is modelled on the Raytheon Phalanx-1B and Thales Goalkeeper systems traditionally fielded by western navies as a last layer of defence against anti-ship missiles (ASMs). These systems have been adapted to operate in a land environment and have proved effective in mitigating the threat from mortar and rocket fire. Information regarding modifications to the Phalanx or Goalkeeper systems for adaptation to land operations was unavailable, thus the following discussion of their application to counter rocket and mortar fire is predicated on their original naval specifications. The weapon engagement system is composed of three individual sensor systems, each designed to perform a separate function within the interception process:

- A pulse-Doppler radar responsible for search, detection, track and target classification. The radar provides a long range, continuous search capability and is capable of maintaining a large number of track files concurrently, prioritising targets based on a range of criteria that incorporate the range, range-rate, bearing and altitude measurements to the target together with prior knowledge of weapon performance. Algorithms suitable for radar target-tracking are discussed in the analysis of the weapon location radar presented in the Detect section of this section. Angle tracking may be improved through the use of a monopulse radar design, which also has the additional benefit of making radar jamming more difficult.

- Dedicated pulse-Doppler, MTI-processing tracking radar designed to engage threats determined by the system's search radar. The tracking radar takes its cure from the search radar, engaging a limited number of priority targets, allowing the system to continue to scan the surrounding area while engaging incoming fire. The angle tracking performance of the radar needs to be especially good in order to accurately direct cannon-fire onto incoming munitions, maximising the probability of a successful kill. The use of a monopulse radar design and appropriate choice of a high-frequency waveform and antenna design would support this goal. The high angular $\&$ range resolution of the tracking radar enables it to track outgoing cannon fire and calculate the "miss distance' from the incoming projectile, allowing the weapon system to correct its angle of fire and effectively steer cannon fire onto the target.

- A Forward Looking Infrared (FLIR), $8-12 \mu \mathrm{m}$ thermal imaging device that compliments the search radar to enhance target detection and identification performance or additionally may be used in a stand-alone mode to 
autonomously track \& engage ground based targets or low-flying aircraft, such as helicopters or UAVs. The FLIR system is capable of outputting a digital video stream that may be connected to a standard TV or computer monitor display to allow an operator to manually assess \& engage ground based targets if required.

The three sensors work in unison to drive a high-speed, high-precision gun-control mount that houses a $20 \mathrm{~mm}$ or 30 $\mathrm{mm}$ cannon (the latter offers greater mass and hence greater stopping potential). A fire-control station accompanies the gun-mount and contains a separate computer system that serves as a control station for a human operator. From this station the operator may define rules of engagement, such as defining the minimum and maximum target velocity, whereby any target tracked by the system that lies outside of those bounds should not be engaged. The operator may also employ zonal control, as discussed previously in the design of the rocket and mortar detection radar in the Detect section of this section, to restrict the operation of the system to a limited area, reducing the workload of the search and track radar systems. The operator station is hard-wired to the gun mount and serves as the sole means of integrating external systems with the CIWS platform. Unit mobility is achieved by mounting the gun mount and fire control station on a large articulated trailer, allowing the unit to be redeployed quickly with minimum setup and teardown time.

\begin{tabular}{|l|l|}
\hline Search Radar & \\
\hline & Ku Band, digital MTI \\
\hline & 6.0 km Maximum Range \\
\hline & 4.3 km Target Acquisition Range \\
\hline & $25 \mathrm{~m}$ range resolution \\
\hline & $2^{\circ}$ angular resolution \\
\hline Track Radar & Ku Band, pulse Doppler monopulse \\
\hline & $4.5 \mathrm{~km}$ Maximum Range \\
\hline & 10 m range resolution \\
\hline & $\sim 1^{\circ}$ angular resolution \\
\hline FLIR Thermal Imager & \\
\hline & $8-12$ micron, CMT CCD detector \\
\hline & $\sim 100$ microradian angular resolution \\
\hline & Automatic Acquisition Tracking \\
\hline & Integrated Digital Processing for image enhancement \\
\hline & Digital Video Out \\
\hline Weapon System & \\
\hline & 30 mm cannon \\
\hline & 2000 round magazine \\
\hline & Fire Rate: 3000 rounds per minute \\
\hline & Muzzle Velocity of 30mm round \\
\hline & System Reaction Time: 3 seconds \\
\hline Integrated Fire Control Station & Maximum probable kill @ 500m \\
\hline Combined Mass & 6 metric tonnes, housed on mobile trailer for quick redeployment \\
\hline
\end{tabular}

Table IV-5 Main features of cannon-based close-in weapon system

The diagram below, Figure IV-5Error! No bookmark name given., presents a functional diagram of the final weapon system for interception of incoming rocket and mortar fire. The system operates as follows:

- Track files from the short-range rocket and mortar radar are relayed via a wireless or wired connection to the fire control station of the cannon-based CIWS. The fire control station is the primary interface between the cannon, sensors and human operator controlling the system, where the tracking data from external systems is first correlated with the current tracks generated by the CIWS system.

- The fire control computer will cue the cannon upon assessing the projectile as an imminent threat. The system will confirm the detection of incoming fire to the operator at the fire control station and recommend the target be engaged. The system will not operate in automatic fire mode due to the high potential for fratricide and collateral damage, requiring manual confirmation from the operator. The operator is also 
capable of viewing video generated from the system's integrated FLIR, allowing human assessment in situations where the system may be tracking low-flying UK aircraft flying in and out of the base. Once authorised, the system will continue to intercept all incoming projectiles without further operator instruction.

- Tracking data from the external rocket and mortar radar and CIWS tracking radar is fused to reduce the uncertainty in a projectile's location and increase the probability of a successful engagement. A separate Kalman filter instance is created for each target tracked by the system for fusing data from external systems.

- The CIWS search and track radar is considered to be adequate for tracking conventional rocket and artillery fire but its performance against mortar fire is unreliable since the radar design lacks the required functionality to reliably detect \& identify the relatively slow-speed mortar. Failure of the external rocket and mortar radar would therefore reduce the capability of the final indirect fires countermeasure system and increase the threat to the base.

- Upon confirming the threat has been neutralised, the system selects the next target posing the highest threat and slews the cannon to the appropriate bearing in azimuth \& elevation.

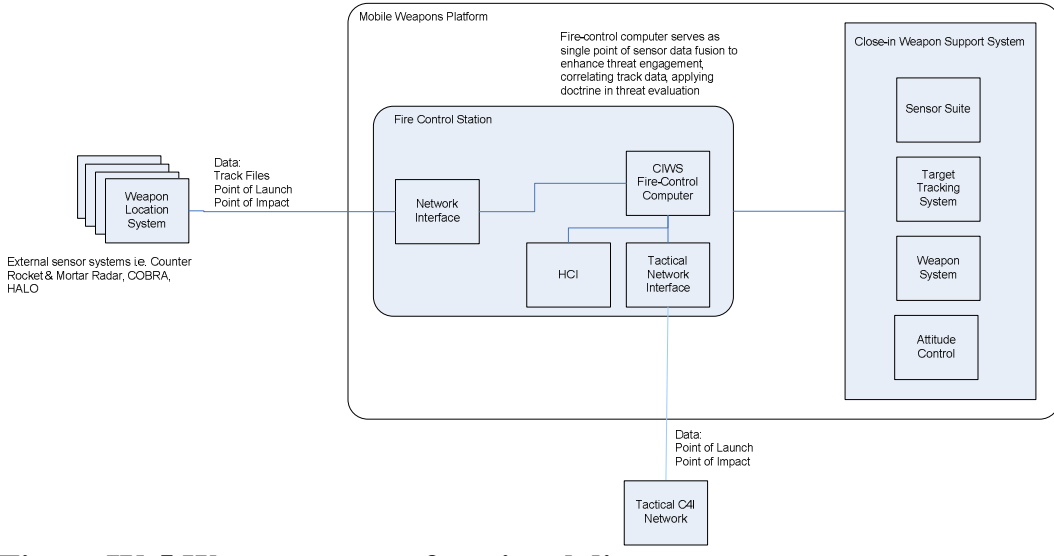

Figure IV-5 Weapon system functional diagram
FigureIV-6 demonstrates one possible deployment scenario for the indirect fire countermeasure system. Given the proximity of the nearby town and hillsides the commander assesses that these are the most likely locations from which militia will attempt to launch attacks on the base and deploys two CIWS systems accompanied by rocket and mortar weapon location radars at opposite corners of the base. The search area of the CIWS and mortar radars is restricted using zonal control via the operator terminals

at the separate fire control stations, restricting the search area of the radars to the area external to the base.

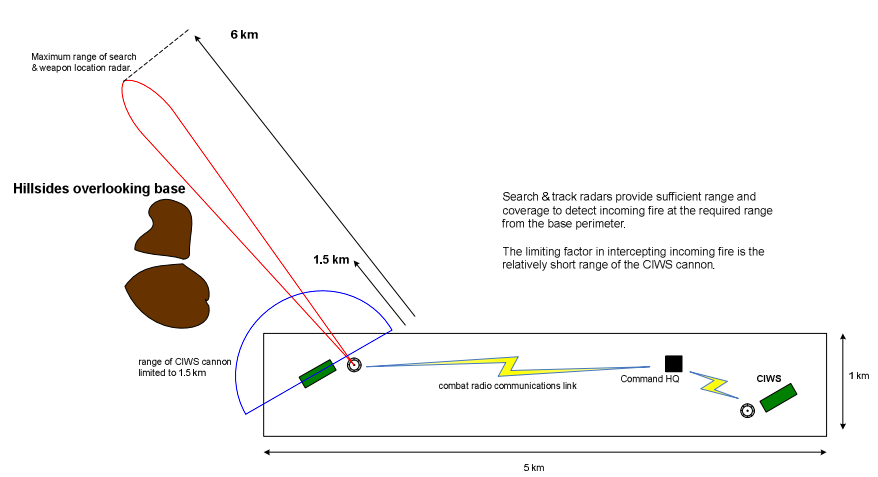

Figure IV-6 Deployment of indirect fires countermeasure system sensors
The determining factor in the effectiveness of the system is the $1.5 \mathrm{~km}$ engagement range of the CIWS cannons, which provide a limited protective screen confined to a small areas at the opposite ends of the $5 \mathrm{~km}^{2}$ base. Further work is required to identify the optimum number \& positioning of indirect fire counter measure systems to provide sufficient coverage and confidence against incoming fire. However, given that the land commander cannot guarantee that he will always have access to sufficient resources in the course of his mission then the deployment of such systems will tend towards protecting the most valuable locations throughout the base as opposed to trying to guarantee continuous $360^{\circ}$ coverage.

\section{Command \& Control}

The Point of Launch (PoL) data generated by the rocket and mortar weapon location radar may be analysed over time to identify the most likely locations around the base from which militia have been found to launch attacks. The use of statistical techniques, neural networks or traditional methods from operations research may be applied in the 
development of an automated system capable of extracting trends and patterns in enemy behaviour from the PoL and Point of Impact (PoI) data. By using the inferences generated by such a system, the commander is capable of tasking his ground patrol or tactical UAV units to deter such threats or quickly responding to an attack. Because the command HQ is integrated with the tactical C4I network, he is able to quickly order nearby forces to an estimated point of launch, greatly reducing the time to intercept militia forces. The closed-loop operation between sensor and ground units is important in dealing with so called 'shoot-and-scoot' attacks that will likely originate from the nearby town.

\section{Summary}

The Indirect Fires counter measure system combines a short range radar dedicated to tracking incoming rocket and mortar fire to a maximum range of $6 \mathrm{~km}$. This man-portable system is integrated into a trailer-mounted, close-in weapon system (CIWS) incorporating separate search \& track radars integrated with a FLIR imaging device, where the sensor data from the two systems is fused at the CIWS fire-control station to provide targeting data to a $30 \mathrm{~mm}$ cannon with an effective range of $1.5 \mathrm{~km}$. Each fire-control system is integrated via the tactical C4I network to the command HQ located within the base, relaying estimated Point of Launch (PoL) and Point of Impact (PoI) data to the command HQ where a dedicated warning system alerts on-site personnel of incoming fire. The fire control system acts as the primary interface to which external sensor systems are integrated to cue the weapon system, where systems such as the Selex HALO or European COBRA system can be integrated in future to provide a layered defence to rocket, mortar and artillery fire.

\section{Perimeter defence}

The following subsections looks at the overall perimeter defence system. It is felt that the perimeter defence aspect is the primary defence system for the base. The system must be able to provide constant surveillance of the local environment and in all weather conditions.

25. Perimeter Surveillance Subsystem Analysis

The perimeter surveillance system is required to provide continuous observation of the area surrounding the operational base to detect and identify potential threats, such as men and ground vehicles, improving the ability of commander to direct his forces to intercept or deter such threats.

The initial system requirements presented earlierwere reviewed to determine those requirements applicable to the perimeter surveillance subsystem and have been expanded after further analysis. These requirements, listed in Table IV-6 below, serve to steer the evaluation of the perimeter surveillance system.

\begin{tabular}{|l|}
\hline The system must provide effective $360^{\circ}$ surveillance beyond the surrounding perimeter. \\
\hline The system must detect men at a maximum range of $3 \mathrm{~km}$ beyond the base perimeter. \\
\hline The system must recognise men at a maximum range of $1 \mathrm{~km}$ beyond the base perimeter. \\
\hline The system must detect ground vehicles at a maximum range of $4 \mathrm{~km}$ beyond the base perimeter. \\
\hline The system must recognise ground vehicles from a maximum range of $2 \mathrm{~km}$ beyond the base perimeter. \\
\hline The system must obtain measurements of target range to a resolution of $20 \mathrm{~m}$. \\
\hline The system must obtain measurements of target bearing in both azimuth and elevation to an accuracy of $4^{\circ}$. \\
\hline The system must display the estimated range \& bearing to a detected entity to a human operator. \\
\hline The system must supply target classification data to the human operator to aid their threat assessment process. \\
\hline The system must provide an effective day and night surveillance capability. \\
\hline
\end{tabular}

Table IV-6 Refined functional requirements for the perimeter surveillance system

\section{J. Candidate Sensor Technologies}

This section provides an overview of the individual threats to the forward base together with an initial set of sensors capable of observing specific attributes of the individual entities. This section reviews those sensor technologies appropriate to the detection of men and ground vehicles to select a sensor(s) that complies with the functional requirements presented in Table IV-6 and are believed to best support the operational context for this investigation.

\section{Radar}

Radar is a long-standing system employed by land forces to estimate the range, azimuth, elevation and velocity of enemy forces such as men, ground vehicles, low flying aircraft and artillery fire. The battlefield surveillance radar 
(BSR) is the most relevant example of the application of radar to countering threats from land forces at the close range $(\sim 5 \mathrm{~km})$ envisaged within this investigation. The advantages of radar include:

- Wide area surveillance capability

- High data-rate, providing large volume of measurements for decision making

- All-weather, day \& night sensor

- Basic capability to detect hidden targets e.g. men or vehicles attempting to use natural vegetation such as trees, bushes to camouflage their presence.

- Single sensing mechanism capable of providing measurements in range, azimuth, elevation and velocity.

- Advancements in miniaturisation and battery technology enable development of a man-portable, quickdeploy surveillance capability.

The disadvantages of radar for perimeter surveillance include;

- Active sensing, rendering the operator vulnerable to DF and ECM.

- Requires sophisticated processing to reduce the effects of ground clutter to ensure sufficient radar range and low probability of false alarm.

- Target classification can prove extremely complex due to nature of radar backscatter, making reliable classification of ground vehicles or low-flying aircraft i.e. helicopters, UAVs difficult.

\section{Electro-optic/Infrared Imaging}

The advantages of EO/IR sensors for perimeter surveillance include:

- Wide area surveillance capability

- Passive sensor

- Capable of producing frequent, high-resolution imagery for target-classification

- Large amount of information present in sensor output for decision making e.g. intensity, shape, colour, contrast, frequency content.

- Raw sensor output i.e. an image, is capable of being interpreted by a human operator and can potentially reduce the requirements on sensor data processing to deliver accurate object recognition.

The disadvantages of EO/IR sensors for perimeter surveillance include:

- Sensor performance can degrade considerably through atmospheric absorption in adverse weather conditions e.g. fog, rain.

- Depending upon desired image resolution, communication requirements can become significant, requiring high bandwidth communications links or, if low bandwidth bearers are the only alternative, the resulting data transfer is subject to high latency.

- No range information available from a single EO or IR sensor.

\section{Acoustic \& Seismic Sensing}

The advantages of acoustic and seismic sensors for perimeter surveillance include:

- Passive sensor

- Capable of classifying targets based upon frequency analysis of acoustic and seismic signals

- Advances in miniaturisation imply small, disposable devices could be deployed in large numbers to provide adequate sensor coverage. Individual sensor nodes could easily be hidden or camouflaged to provide a concealed sensor system for base protection.

The disadvantages of acoustic \& seismic sensing include:

- High levels of background noise and audio 'clutter' from and the presence of a nearby urban centre provide a challenging sensing environment.

- A single acoustic or seismic sensor is not capable of providing sufficient coverage of the area surrounding the operating base, requiring a network of distributed sensors. Deployment of the sensor network to provide adequate coverage is both complex and time consuming.

- A distributed network of unattended ground sensors surrounding the base may require continuous repair if subject to regular attack from mortar fire, requiring the commander to retain resources for deploying additional sensor nodes to maintain adequate coverage and detection performace. 
- Sensor node density, that is the number of sensor nodes within a given area, has a significant impact on the underlying communications and computing infrastructure. A sensor network with high node-density will require significant computing and communications resources to process high-volumes of data.

- The operational lifetime of the system is limited by the capacity of batteries necessary to power each sensor node.

Having considered the potential of each type of sensor for application within a perimeter surveillance system it is recommended that EO/IR sensors be further analysed to determine their ability to meet the supplied system requirements. None of the additional sensors highlighted above will be considered for application within a final design as it is believed that the sole combination of EO \& IR sensor technology is adequate. Radar is capable of measuring range, bearing and target velocity in varying weather conditions, yet the data it provides by itself does not make it easy to determine what is and is not of importance from the sensor returns i.e. a ground vehicle was detected $2.3 \mathrm{~km}$ from the base perimeter, but is it friendly, hostile or civilian? In contrast, EO \& IR sensors deliver high value data better suited to address such concerns due to the ability to detect, track, recognise and identify entities from sensor returns and may also be analysed by human operators to resolve ambiguities that may arise.

\section{EO/IR Ground Target Surveillance}

The perimeter surveillance system employs a conventional CCD colour day camera coupled with a separate uncooled $8-12 \mu \mathrm{m}$, IR imaging device (FLIR) to provide day \& night observation of the area surrounding the forward base. The EO \& FLIR systems are capable of obtaining a 'static' image or a live video feed containing targets of interest that can be processed to extract the bearing to the target (when knowledge of the unit's attitude is known) or be presented to a human operator for further analysis. The performance of the EO/IR surveillance camera is loosely modelled on a TI surveillance system available from Qioptiq Group ${ }^{18}$ known as DAGIR and serves as an indicator of the likely performance of current optical systems. The table below, Table IV-7, lists the technical specifications of the EO/IR surveillance camera, including the range performance of the imaging system defined according to the Johnson criteria;

\section{Day Camera}

IR detector type
IR detector resolution
Field of View
Full pan, tilt \& zoom control
Combined Mass

Range Performance (Clear Air)

Detection

Recognition

Identification
2D 'staring' CCD

$640 \times 480$ line resolution

26x optical zoom

uncooled, $8-12 \mu \mathrm{m} 2 \mathrm{D}$ CCD

$640 \times 480$

4.30 narrow / 12.80 wide

$10 \mathrm{~kg}$

Table IV-7 technical specifications of the EO/IR observation system

The high resolution of the IR detector was deliberately chosen to maximise the range performance of the system and to supply television-quality video to a human operator for further analysis of entities at suitably close range for identification. The latter feature of the sensing system implies a requirement for a high-bandwidth communications link to transmit the potentially large images to a designated host in the $\mathrm{C} 2$ network. The detector is also of an uncooled type, reducing the mass, power and maintenance requirements of the system over a cooled, photon-based IR detector. To provide adequate coverage of the surrounding area in both range and azimuth the camera system is mounted onto a mast or small tower to elevate the system above the surrounding buildings and walls of the base. The elevation of the camera makes it susceptible to vibration from high winds and thus requires stabilisation in order to minimise distortion or blur in the image produced across the detector. The tower upon which the camera is

\footnotetext{
${ }^{18}$ http://www.qioptiq.com/
} 
mounted with a GPS receiver in order to accurately determine its geolocation and the EO/IR surveillance unit is integrated with a basic Inertial Measurement Unit (IMU) in order to track the orientation and elevation of the camera. The location and orientation of the camera serves as metadata that may be encoded into an image or video stream that, when processed, can be used to estimate the geo-referenced location of entities within the area observed by the camera system. Since the EO/IR surveillance unit is limited to measuring the relative bearing of a target within its field of view the unit is integrated with a laser range finder (LSR) to provide highly accurate estimates of the relative range of the target from the camera's location. Note the LSR is boresighted to the EO/IR unit, ensuring accurate range estimates of objects detected within the camera's field of view, and operates at an eye-safe wavelength within the infrared spectrum, rendering the laser operation invisible to the naked eye.

\begin{tabular}{cc} 
laser wavelength & $1.5 \mu \mathrm{m}($ Er:Glass $)$ \\
minimum range & $300 \mathrm{~m}$ \\
maximum range & $7.0 \mathrm{~km}$ \\
range resolution & $\pm 10 \mathrm{~m}$ \\
\hline
\end{tabular}

Table IV-8 Technical specifications of the selected laser range finder

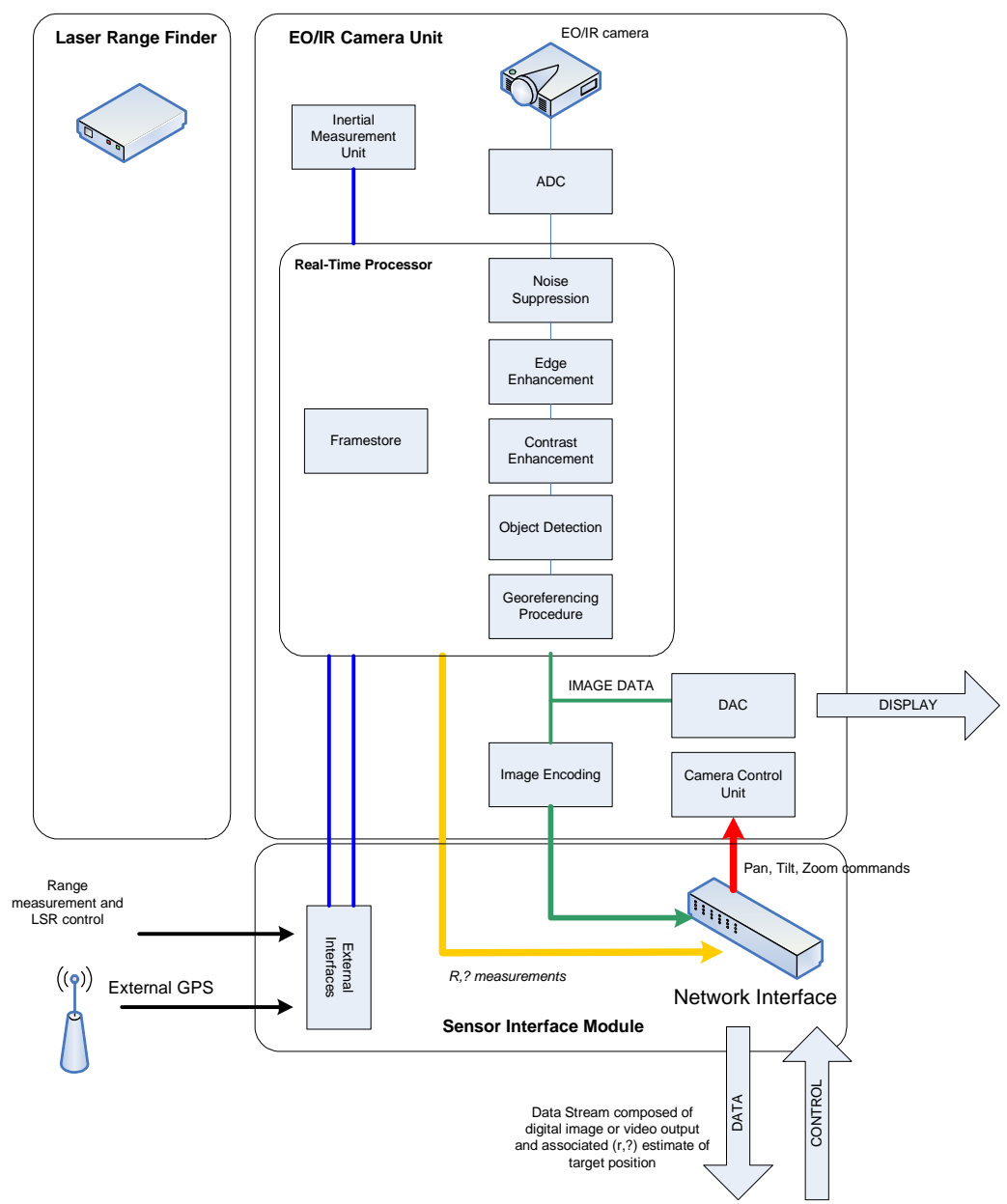

Figure IV-7 Functional architecture of individual surveillance sensor node
The Figure IV-7, illustrates the various functional elements that comprise a sensor node in the perimeter surveillance system. The raw output of from the daylight \& IR camera is first digitised and the current output from the GPS and IMU systems sampled and stored together with the resultant frame. The output of the daylight and IR systems will be subject to noise, blur or vibration that corrupts the image and reduces the overall ability to detect, recognise and identify entities within the camera's field of view. To counter image corruption, each frame is processed in real-time to remove the effects of noise, blur and improve the contrast of the final image via a set of convolution filters. Conventional computing architectures, despite their current level of advancement, are inappropriate for delivering adequate real-time performance for image or video (24 fps) processing, requiring an FPGA or ASIC-based solution capable of enhancing every individual frame within $1 / 24 \mathrm{~s}$ or less. Following image enhancement, the image frame is rapidly scanned for any possible objects of interest (for TI output this involves a rapid test for 'hot' pixels with the application of a simple binary threshold, based on prior knowledge of the likely intensity distribution of targets of interest, to separate out the target from the background). Objects detected within the field of view may be the result of glare or optical 'clutter' requiring further temporal and spatial processing of sequential image frames to confirm the presence of an entity. If an object is finally detected within the camera's field of view, the relative bearing to the target is computed from the current frame and the LSR fired to estimate the relative range to the target. The relative location of the detected object may be combined with the values of the camera's attitude and the mast's geo-location 
to geo-reference the target, where the final output of the camera is an enhanced image \& accompanying set of georeferenced locations for objects detected within that image. If no object is detected within the camera's current field of view then the camera system may be configured to simply drop the current frame since it contains no additional information, thus reducing the load on the supporting communications infrastructure and any downstream processing.

\section{System Integration}

System integration addresses the bringing together of the EO/TI surveillance unit with communications, computing and HCI components to deliver a complete system capable of acquiring and exploiting sensor information. The primary challenges to the successful integration of the EO/TI surveillance unit are:

- The transfer of high-resolution imagery/video captured by the daylight \& TI camera to a distant node in the communications network for further analysis.

- Developing a deployment strategy for individual surveillance units that meet the range and coverage requirements for the perimeter surveillance system. The placement of the sensors will further influence the requirements on the communications network to deliver sensor data to a target unit or platform.

- The availability of systems and procedures that reduce the workload of an operator charged with monitoring and exploiting the output from multiple EO/TI surveillance units located throughout the base. A human operator has a limited capacity to continuously monitor the outputs of multiple video streams and will eventually tire. The use of a large number of analysts merely increases the manpower requirements of operating the surveillance system; the system is required to reduce the resources required for maintaining effective base security.

- Minimising the delay between 'sensor-to-soldier' to deliver $360^{\circ}$ surveillance of the surrounding area in realtime.

\section{K. Candidate Perimeter Surveillance System Solution}

The diagram below, Figure IV-8, illustrates a proposed architecture for a wide-area perimeter surveillance system capable of detecting men and ground vehicles surrounding the forward base. The integrated system provides the commander with the capability to:

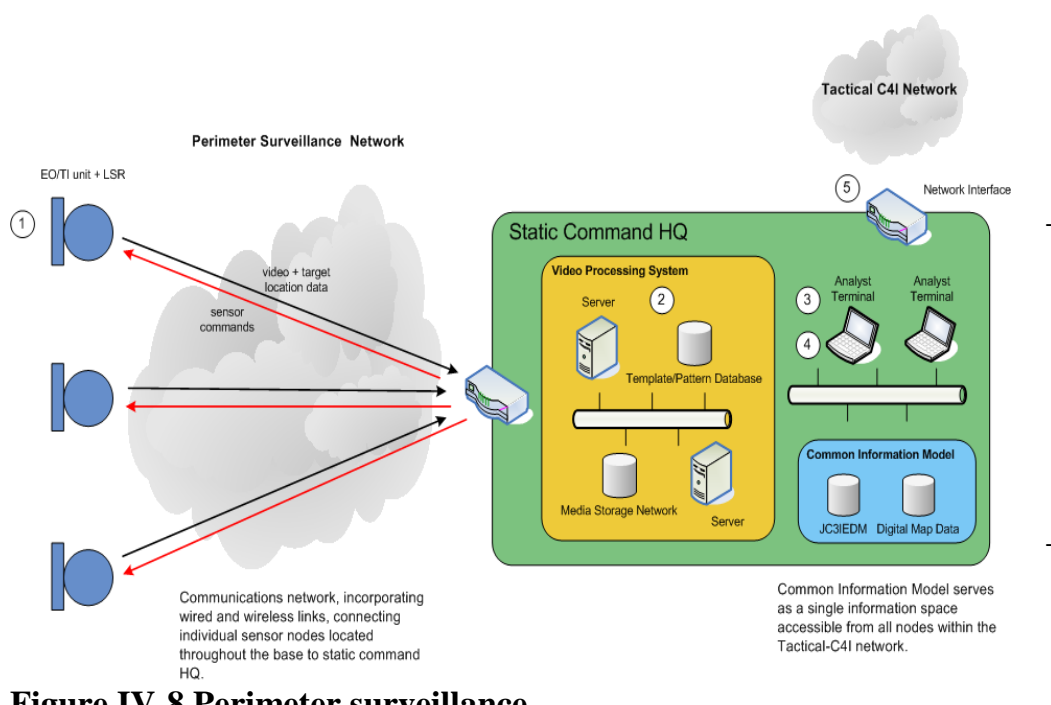

- Detect entities approaching the operating base from any direction and with suitable warning so as to direct his forces to intercept or deter such forces from approaching any further.

- Reduce the number of personnel required to operate the surveillance system by reducing the workload of human operators monitoring multiple video streams for potential threats via the provision of an automated target identification and recognition system.

- Distribute the estimated position of entities detected by the perimeter surveillance system to mounted and dismounted units patrolling the surrounding area via integration with the tactical C4I network.

1. Video, imagery and targeting data produced at each individual surveillance unit is relayed back to a dedicated installation within the command HQ for further processing and archiving via a point-to-point communications link. Where sensor deployment renders a wired connection impractical then a wireless communication bearer will be required to relay the data stream. The high-resolution of the IR and daylight cameras coupled with the high-frame rate (minimum $25 \mathrm{fps}$ ) for PAL-equivalent video transmission requires a high-capacity communications link to relay sensor output with sufficiently low latency for real-time analysis of imagery and video. The communications system employs encryption, rendering the data stream incomprehensible if 
intercepted by the enemy. An analysis of the requirements on the communications network, and options for reducing the load on the network is presented below in Network Performance.

2. At the command HQ the video \& image streams from each surveillance unit are stored and processed by a dedicated series of image processing workstations to perform automated recognition \& identification of objects within a scene. This video processing system employs a combination of COTS \& dedicated hardware optimised for processing multiple video streams concurrently and has access to a database of known templates and patterns related to targets of interest that are applied in the target identification and recognition process. Suitable techniques to support this process include the use of Fourier descriptors and statistical moments, both techniques that are tolerant, to a degree, to variations in object aspect \& rotation. Targeting data associated with each stream is also saved stored alongside the video feed and is made available for existing situational awareness applications employed by the commander and his staff.

3. A target of interest confirmed by the system generates an immediate alarm that is sent to an appropriate analyst together with the current location of the target. The location of the target and its class (man, vehicle) is presented as a symbol, representing an unconfirmed target, on a digital map display that forms part of the analyst's situational awareness application. The analyst is capable of requesting the video/imagery associated with that target from the video-processing system where it is displayed immediately on their terminal, allowing the analyst to monitor the feed and confirm the target is hostile or is of no concern. Objects of interest within the displayed scene are annotated with the automated processing systems estimate of the target type, drawing the attention of the appropriate area of the screen.

4. The analyst is always capable of selecting a real-time feed from any individual sensor at any time and is thus not dependent upon the automated video processing system in order to be made aware of activity around the base. Furthermore, the control system for the cameras is integrated with analysts digital map display, allowing the analyst to mark areas of interest on the map that are then converted to commands controlling a surveillance unit. This allows the analyst to adapt system behaviour to mission requirements, where focus may be directed to specific areas around the base if $360^{\circ}$ surveillance is not required.

5. Upon confirming a target detected by the perimeter surveillance system is indeed a valid threat, the analyst is capable of publishing the target location data into a Common Information Model accessible to all units within the tactical C4I network. Providing a radio bearer with sufficient bandwidth is available, a still image of the detect target may also be transferred to patrolling units via the tactical C4I network to aid them in recognising the target.

\section{Network Performance}

The communications network linking the surveillance units positioned throughout the base are required to relay a video stream from each unit to a central point (the command HQ). The following calculation, Equation IV-1, provides an example of the data-rate required to transmit a 1-second sample of uncompressed PAL-colour digital video if no compression were to take place:

$640 \times 480 \times 24$ bits per pixel $=7372800$ bits per frame

To transmit 1 - second of PAL video $=7372800 \times 25 \mathrm{fps}=184320000 \mathrm{bits}$

$$
\approx 180 \mathrm{MBs}^{-1}
$$

\section{Equation IV-1 Calculation of uncompressed RGB data transfer rate for PAL-video}

Clearly this is a very large amount of data and is compounded by the need to support additional feeds from multiple sensor nodes. It is unrealistic (and uneconomic) to develop a communications system to handle uncompressed digital video thus we require a means to reduce the load on the communications network. Compression is the first step in reducing the data transfer rate to ensure the use of digital video is practical. Video and image compression seeks to exploit spatial and temporal redundancy in imagery, where compression techniques may be split into two classes: lossy and lossless. Lossless compression techniques offer the advantage of reducing the size of video data without any data loss and retaining maximum image quality; however such techniques cannot achieve the same compression rates as lossy-compression. In contrast, lossy compression can achieve higher compression rates but does so by discarding redundant data that inevitably reduces the quality of the final compressed image. Which compression strategy to adopt will depend upon the level of quality in the final image required by the automated video processing algorithms in order ensure accurate target recognition \& identification with suitably low false 
alarm rates. A second strategy for lowering the load on the communications network is to exploit the fact that the human analyst does not need to monitor a live video feed from every single surveillance unit to identify potential threats since to do so can overload the operator with information. We can therefore command each surveillance unit to only transfer a live video feed when requested by the analyst. Furthermore, the automated target recognition \& identification system located at the command HQ does not require a full 25 fps frame rate in order to classify potential threats within a camera's field of view; the surveillance unit can be commanded to transfer video at a much lower frame rate, say $5 \mathrm{fps}$, and still provide the automated target classification system at a sufficient rate so as to allow it to attempt identify potential threats with confidence. Upon flagging a potential threat from the particular camera feed, the analyst may then request a full 25 fps video feed to confirm the target is indeed a threat.

\section{Complementary Sensor Technologies for System Evolution}

In addition to the above system components there are complimentary systems that can be added in the future to enhance the overall effectiveness of the system. One main system that could be incorporated is a battlefield surveillance radar. This will be able to supply data on targets approaching the base from distance, poor weather surveillance and aid in the manual guidance of munitions onto a target providing feedback on impact zones.

\section{Integrated Solution}

When integrating many disparate systems there are numerous aspects that need to be considered such as using centralised or decentralised processing and fusion, what data storage will be employed and what system redundancy will there be. These are just a few of the areas that will be looked at in this section. The sensor systems that have been designed in the previous section are to be brought together to produce on large coherent system to be used to defend a brigade-style base from the previously named threats. It is envisaged at this stage that the systems will be able to collaborate with each other, where required, and also to provide sufficient data so that a suitable situational picture can be produced. The resulting information will be used to support the commander's decision to either attack or monitor potential targets and also to help focus the sensors on specific tasks as required. The following subsections look at the final solution logical architecture. The $\mathrm{C} 2$ logical block is decomposed further to highlight the internal interactions the external systems have with each other, the data storage capabilities and finally the data fusion techniques that are used to exploit the data collated. The sections will also include a small introduction to the fusion model employed along with some discussion on the data storage techniques employed. Finally the data dissemination requirements of the system will be highlighted. There is no aspiration to select a database, data structure or specific fusion technique within this project, only to highlight the options that will be available and the likely fusion techniques that will be employed.

\section{Solution Architecture}

This subsection looks specifically at the logical architecture created. It brings all of the disparate and secondary sensor systems together in an attempt to create a synergistic system that is able to provide reactive information to the commander. The diagram below, Figure V-1, shows the logical architecture. All of the subsystems that are shown have been described in the preceding sections. As can be seen in the above diagram, Figure V-2, all of the systems converge on the main $\mathrm{C} 2$ system. This is where all the information will be stored and acted upon. This said there will be some fusion and, where applicable image manipulation performed locally at the sensor, when required. The diagram below, Figure V-2, shows the high-level logical architecture of the C2 node.

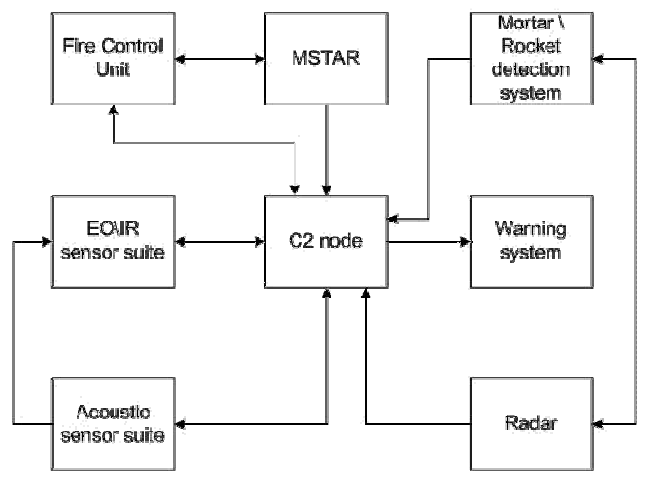

Figure V-1 Logical architecture

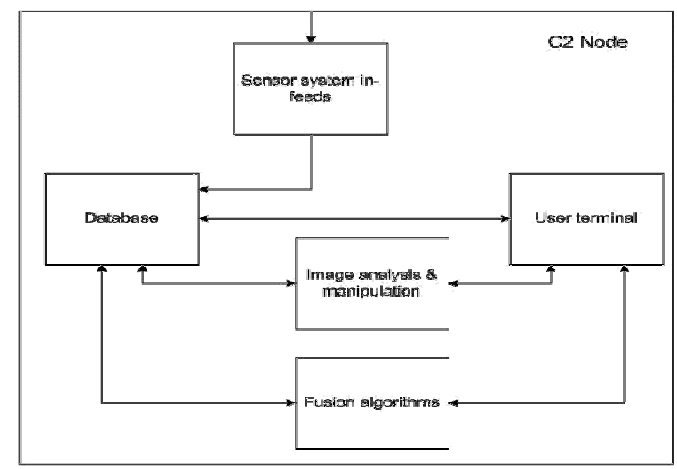

Figure V-2 High-level logical architecture of C2 node 


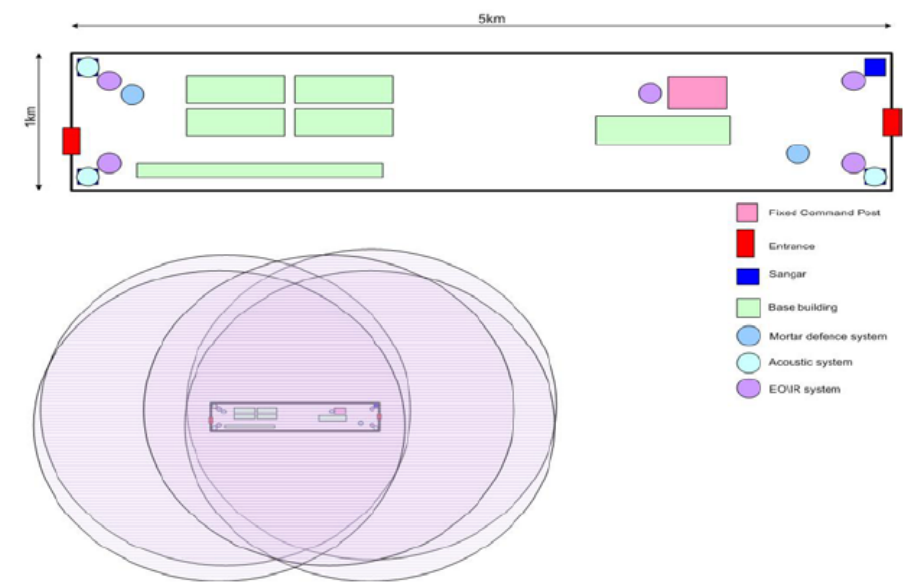

Figure V-3 Sensor system placement and effective primary

The logical architecture can now be translated into the base lay down in order to ensure the required connections can be achieved. In the diagram above, Figure V-3, can be seen the chosen configuration of the base (the top image) and the effective coverage range of the main base sensor suite (bottom left corner). In each corner of the base there is an EOUR sensor component. There is also a component collocated with the fixed command post. As there is a mountainous region to the west of the base and a town to south and south east of the base the acoustic sensors have been strategically placed leaving the north eastern corner free; it is deemed that there are likely little attacks from snipers from an open desert position. There is a mortar detection system placed to the east and west of the base providing protection from potential attacks from the town and mountainous zones. The main gate to the base is to the east and the secondary gate to the west. These two zones are well serviced by the primary sensor system, the EO \& IR system, and the sniper detection system. Considering the image showing the coverage of the main base sensor system it can be seen that the system does provide enough coverage for the short range defensive aid suite. The links to the main $\mathrm{C} 2$ system will consist of both wired and wireless links. At the extremities of the base, where the visual and IR systems reside along with the acoustic sensors and mortar detection system it is likely that these will be connected via wireless links. It is expected that these links will be high capacity data links capable of transmitting imagery at a rate suitable enough to provide the information the commander requires. The main visual system, which is collocated with the $\mathrm{C} 2$ tent, will be hard wired into a terminal in the tent providing the commander with a live, real-time feed. This will be under the control of the commander directly. Where sensors have secondary systems collocated, such as the EOIR sensors near to the acoustic sensors, these connections will also be hardwired. There will be the capability for the soldier at the outpost to view only the data supplied to that outpost. Data from that outpost will be subscribed to by the commander and delivered accordingly. As the location of the sensors has been chosen and the relevant communications link provide it can be seen that there should be little issue in passing data across the base network. There will undoubtedly be the standard voice terminals available along with any other required data terminals to display situational awareness information however this is not part of the system under design here. Two major aspects of the design that were considered throughout the design process were redundancy and scalability. If a sensor system should fail then there needs to be either a back or short term cover until the main system returns to full capability. The natural choice in this scenario would be to have multiple back-up systems however factors such as cost and logistics mean that you cannot simply throw more tools at the problem. The following table, Table V-1, highlights the system, what provides cover should the system fail and finally the aspects (issues) that are not covered by the covering system.

\begin{tabular}{|l|l|l|}
\hline Main System & Covering System & \multicolumn{1}{c|}{ Issues } \\
\hline Acoustic & EO \IR & $\begin{array}{l}\text { Relies on camera staring at the muzzle flash in } \\
\text { order to obtain detection. }\end{array}$ \\
\hline Mortar & EO \IR & $\begin{array}{l}\text { Relies on the camera focusing on the mortar as } \\
\text { soon as it has been fired. }\end{array}$ \\
\hline EO \IR & Radar & Doesn't provide any visual data to the end user. \\
\hline
\end{tabular}

Table V-1 Redundancy throughout the system

It must be noted that although the covering sensors identified can provide a form of coverage extra functionality and consideration would be required to make them perform as good as the primary sensors in these areas. There is a level redundancy built into the system however it is never possible to create full redundancy of capability unless endless versions of each system are available. What this does highlight though is the absolute requirement for a system to be down for the minimum amount of time possible, if at all. As each sub system was designed scalability was always a factor that was considered. Looking at the EO \IR system, as an example, it can be seen that it consists 
of both wired and wireless linked components. The system can also be employed as a secondary sensor system used to gather more data on potential targets. The system can grow, with further inclusions of cameras. The subsequent data placed on the network will be small as the aim of the system is to perform the majority of the processing and fusion at the sensor and pass only the required minimal data set to allow a commander to make an informed decision. As mentioned above, the various systems employed can double up as secondary sensors to other systems. This allows for reuse of sensor systems subsequently reducing the overall cost and logistic pressure.

\section{Functional Fusion Model}

Regardless of the size and complexity of the system there should always be, in some form, a fusion model employed. The model will allow the end user to make more informed decision on the actions to be taken at all levels throughout the battlefield; from company level right through to division level. There has been some work done in trying to ascertain the best methodology to follow

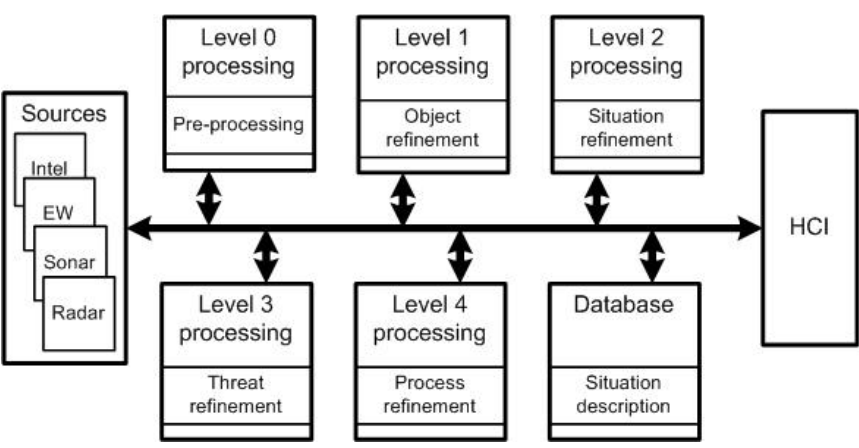

Figure V-4 High level fusion process model when dealing with data from both disparate and cooperative sensor systems ${ }^{19}$. For this project the Joint Directors of Laboratories (JDL) Level 5 fusion model was chosen. The diagram below, Figure V-4, shows the high-level functional model of the data fusion process. The work done by Blasch et al highlights the requirement for some human interaction; this will introduce some user refinement to the overall process allowing for greater accuracy of target localisation. It is assumed that this process will occur as part of the standard C2 standard operating procedures as well as to increase the integrity of the information for use in the strategic outlook.

In summary, as highlighted ${ }^{20}$ in the levels shown in the above model break down to cover the following:

- Level 0 - Sub-Object Data Assessment: estimation and prediction of signal/object observable states on the basis of pixel/signal level data association (e.g. information systems collections);

- Level 1 - Object Assessment: estimation and prediction of entity states on the basis of observation-to-track association, continuous state estimation and discrete state estimation (e.g. data processing);

- Level 2 - Situation Assessment: estimation and prediction of relations among entities, to include force structure and force relations, communications, etc. (e.g. information processing, FDP, FL);

- Level 3 - Impact Assessment: estimation and prediction of effects on situations of planned or estimated actions by the participants; to include interactions between action plans of multiple players (e.g. assessing threat actions to planned actions and mission requirements, DM, PE);

- Level 4 - Process Refinement (an element of Resource Management): adaptive data acquisition and processing to support mission objectives (e.g. sensor management and information systems dissemination, IO, C2).

- The database plays a fundamental part in the whole process. Selecting the right type of database can have a particular influence on the timeliness of the overall process and how inter data relationships, amongst other things, are developed. This topic is covered more in the data storage section later.

The system being designed here has the capability to exploit all levels of the fusion process described above. Level 0 -2 will be predominantly performed at the sensor, especially when considering the likely requirement for the mortar system act with immediate effect upon the incoming munitions.

With the dissemination of data throughout the battlefield to either the main $\mathrm{C} 2$ node or to secondary support sensors further Level 2 fusion can occur. Once the data has arrived at the C2 node there will mainly be Level 3 - 4 fusion performed.

\section{N. Data Fusion Techniques}

${ }^{19}$ Blasch, E and S. Plano, "JDL Level 5 fusion model: user refinement issues and applications in group tracking," SPIE Vol 4729, Aerosense, 2002, pp. $270-279$

${ }^{20}$ Multisensor Fusion By Anthony K. Hyder, Elisa Shahbazian, Edward Waltz 
Data fusion will have already occurred at the sensor in order to allow for minimum data transmission requirements. However data is transmitted to the $\mathrm{C} 2$ node where it can be fused to allow for more information to be obtained, potentially. Although single sensor readings are not necessarily reliable, leading to more sensors being used to provide more accurate readings, in this system there are many sensors employed in each sub system. As such it is felt that fusion at the $\mathrm{C} 2$ node will possibly improve the information, although it will primarily be used to build up a more strategic and operational view of the immediate battle space. The fusion techniques required vary from those used on raw data, techniques such as Kalman filtering, Markov modelling and other such probabilistic techniques. The sensors systems used can be identified as complimentary, competitive or cooperative; the fusion chosen dependant upon the systems under scrutiny. There is much redundant information contained in the data captured from like sensors however when fused with data captured from different sensors more information can be found. The following list looks at the various techniques that will be available for selection in the $\mathrm{C} 2$ node:

- Feature-based inference

- Parametric (Inference, Bayesian, Dempster-Shafer)

- Information-theoretic models (artificial neural networks, figures of merit, pattern recognition)

- Cognitive-based (fuzzy set theory)

- Data alignment

- Data and object correlation

There is no intent to specify the exact fusions algorithms that will be employed as further investigative work would be required to find the most optimal methods to use.

\section{O. Data Storage and Exploitation}

This section looks at the various aspects of data storage and management along with the exploitation of the data that has been harvested. It is not the intention of the following sections to highlight the actual database that will be used as this is deemed to be a implementation decision. However this said it is worth taking stock of the kind of database and data structure standards that are available for use as it is possible for there to be some high level impact on the system design.

\section{Databases and data}

When collating vast amounts of data in differing formats it is vitally important that the data is readable, accessible and easy to use. Prior to selecting the database the type of data structure needs to be decided upon. Selecting the correct data structure will hopefully allow for:

- efficient storage;

- rapid search and retrieval of data;

- regular and set operations to be performed with ease;

- ease in retrieving context sensitive data;

- provide multiple levels of abstraction.

This project is not concerned with the selection of data structures and databases however it is important to show some consideration to the domain when designing a system. As well as looking at bespoke formats, however it is worth considering the more general and standard formats that exist. As such it is useful to consider designing a system to handle structures supported by systems such as LINK 16 for example. Once a suitable data structure has been decided upon, the database of choice needs to be integrated. There are three main types of databases to be considered; relational, file based and object oriented. The main aspects of each system to be considered are storage, search, manipulation and management; the required operational tempo and data to be stored will influence different aspects of the database. Along with this choice there is the decision to have a local database, distributed database or a hybrid of the two.

\section{Exploitation}

When data is to be exploited there are various aspects that need to be considered. Fusion is the obvious area that needs to be considered and the fusion of data at various stages has already been mentioned, however prior to the process of fusion there needs to be some manipulation of the data to ensure it is correctly aligned. In order to correctly fuse data it needs to be aligned in both time and space to ensure the data sets are in a like format. As data is received from the various sensor systems around the base it will be placed into the database with all its associated information. Once called upon the data will need to be geometrically aligned through the use a of a coordinate frame translation algorithm and also matched temporally. There is little useful result of data from different time periods being fused, especially if they are for disparate sensors. Once the data has been fused it needs to be exploited. The information will be required in various forms by various people to ensure they are able to make the best informed 
decision. A such once a choice has been made to act upon a target the correct formatted data needs to be passed to the Fire Control section in order to lay munitions upon a given area. However image analysts may be required to check the imagery in order to spot targets that a normal user may not have otherwise spot. This may impact further decisions. As can be seen there are various destinations for the data and information dependant on the overall operational requirement. One other major aspect of information analysis is the pattern of life. This mainly concerned with establishing trends in data in order to detect various traffic or personnel routes that are regularly used. It also helps to spot potential safe houses that the enemy can use before initialising a strike on the base. This information is invaluable however it takes time to gather in order to create a confident conclusion of activities.

\section{Conclusion}

In this paper we have presented a multi-sensor surveillance system capable of both target detection and target elimination. The system has been specifically designed for threat detection within close range of the base; the type of attack that has become prominent in asymmetric warfare seen in the Middle East today. The distributed nature of the individual systems, and final system, provides a robust and scalable solution. The employment of sensor fusion techniques at the sensor nodes allows for minimal data packets to be sent across the network, allowing sensors to be linked via wireless radios. The internal architecture of the complete system allows for high bandwidth links to support any commander requirements to draw on vast amounts of data when required, for limited amounts of time. The main identified threats; indirect fire, sniper, vehicles and personnel, are all detectable by the systems available, and the redundancy throughout the system means that data, albeit limited at times, can still be obtained from these threats by the systems there. An often lost point that should be made is with regard to the overall scale of the systems designed. It is felt that the overall costs, training, and usability of the systems are reasonable. A main aspect of the system is to be able to reduce the overall number of troops that are required to satisfy the defensive requirements of such a base. It is felt that this system will be able to reduce the number of personnel that are placed in potential life threatening situations as well as allowing the decision makers to better protect the base and the military staff within.

\section{Bibliography}

1 Power to the Edge: Command...Control...in the Information Age - David S. Alberts \& Richard E. Hayes, CCRP Publications

2 Network Centric Warfare: Developing and Leveraging Information Superiority - David S. Alberts, John J. Garstka \& Frederick P. Stein, CCRP Publications

3 Principles of Data Fusion Automation - Richard T. Anthony, Artech House, Inc.

4 Multi-Sensor Fusion: Fundamentals and Applications with Software - Richard R. Brooks \& S. S. Iyengar, Prentice-Hall

5 Jane's Online 